\title{
The Dissipation of Trapped Lee Waves. Part I: Leakage of Inviscid Waves into the Stratosphere
}

\author{
Dale R. Durran, Matthew O. G. Hills, * and Peter N. Blossey \\ Department of Atmospheric Sciences, University of Washington, Seattle, Washington
}

(Manuscript received 14 August 2014, in final form 7 October 2014)

\begin{abstract}
Leaky trapped mountain lee waves are investigated by examining the structure of individual linear modes in multilayer atmospheres. When the static stability and cross-mountain wind speed are constant in the topmost unbounded layer, modes that decay exponentially downstream also grow exponentially with height. This growth with height occurs because packets containing relatively large-amplitude waves follow ray paths through the stratosphere, placing them above packets entering the stratosphere farther downstream that contain relatively low-amplitude waves. Nevertheless, if the trapped wave train is generated by a compact source, all waves disappear above some line parallel to the group velocity that passes just above the source region.

The rate of downstream decay due to leakage into the stratosphere is strongly dependent on the atmospheric structure. Downstream dissipation is often significant under realistic atmospheric conditions, which typically include elevated inversions and strong upper-tropospheric winds. On the other hand, idealized profiles with constant Scorer parameters throughout each of two tropospheric layers can exhibit a wide range of behaviors when capped by a third stratospheric layer with typical real-world static stability. Assuming the Scorer parameter in the stratosphere is a little larger than the minimum value necessary to allow a particular mode to propagate vertically, the rate of downstream decay is more sensitive to changes in the height of the tropopause than to further increases in the stability of the stratosphere. Downstream decay is minimized when the tropopause is high and the horizontal wavelength is short.
\end{abstract}

\section{Introduction}

Trapped mountain lee waves are gravity waves whose energy is largely confined to the lower troposphere. When triggered by long ridges, these waves can produce regularly spaced lines of cloud parallel to the ridge crest. The basic dynamics of trapped lee waves are described in the classic paper by Scorer (1949), who showed that wave trapping is favored when the cross-mountain flow exhibits a sufficient decrease with height in the parameter $N / U$, where $N$ is the Brunt-Väisälä frequency and $U$ is the cross-mountain wind speed. This type of profile can be encountered relatively frequently when the midlatitude westerlies impinge on north-south mountain barriers.

* Current affiliation: Atmospheric Sciences, University of Utah, Salt Lake City, Utah.

Corresponding author address: Dale Durran, Dept. of Atmospheric Sciences, University of Washington, Box 351640, Seattle, WA 98195

E-mail: drdee@uw.edu
Although $N / U$ may be small in the upper troposphere, it generally increases with height in the lower stratosphere owing to the increase in static stability above the tropopause and the decreasing winds above the core of the jet. As a consequence of the increase in $N / U$ in the stratosphere, almost all real-world lee waves can potentially leak energy upward as some fraction of the wave originally launched by the topography propagates vertically through the stratosphere. The possibility that leakage into the stratosphere is an important producer of lee-wave decay is supported by several observational campaigns (Vergeiner 1971; Brown 1983; Shutts 1992; Georgelin and Lott 2001). Trapped-wave leakage has previously been studied theoretically using linear theory in several ways: for deep multilayer atmospheres topped by a boundary condition that prevents upward energy propagation (Corby and Sawyer 1958); for a constant-stability troposphere through which the winds increase with height, capped by a uniform-wind speed stratosphere (Wurtele et al. 1987; Keller 1994); and through asymptotic approximations to the far-field solution generated by an isolated mountain (Berkshire and Warren 1970; Berkshire 1975; Berkshire and Pickersgill 1978). 
Some of these theoretical studies have suggested that, despite the increase in $N / U$ above the tropopause, trapped waves do not leak significant energy upward. For example, Corby and Sawyer (1958), focusing on trapped lee waves "which attain their maximum amplitude in the lower troposphere," state that such a wave "appears without perceptible change in its properties whatever conditions are assumed at high levels ... this is not a fortuitous result arising because of the particular $l^{2}$ profile adopted, but is true in general" $\left[l^{2}\right.$ is defined in (4)]. In contrast, Wurtele et al. (1987) and Keller (1994) both obtain clear evidence of trapped-wave leakage into the stratosphere but do not include systematic investigations of the sensitivity of such leakage to the atmospheric structure.

More recently, the observations analyzed in Smith et al. (2002) led to an alternative hypothesis about the processes responsible of the demise for trapped wavesnamely, that trapped waves were decaying downstream owing to absorption in a stagnant boundary layer. Follow-on studies (Smith et al. 2006; Jiang et al. 2006) have explored how boundary layer processes can lead to the downstream decay of trapped waves. A companion paper (M. O. G. Hills 2015, unpublished manuscript, hereafter Part II) will compare the relative importance of stratospheric leakage and surface friction in producing decay in trapped waves. Distinguishing between these two possible sources of decay is important because it helps us understand whether trapped waves ultimately exert a drag on the flow in the upper atmosphere (owing to the amplification and breakdown of waves leaked upward into regions of ever decreasing atmospheric density) or near the surface (owing to boundary layer processes). Our goal in this paper is to explore the behavior of individual partially trapped modes that may lose amplitude downstream owing to the leakage of energy through the stratosphere and to determine the sensitivity of such leakage to the atmospheric structure. In addition to considering a broad range of atmospheric structures, our approach differs from previous theoretical work through its focus on individual modes-that is, individual solutions to the governing equations with a single (possibly complex) horizontal wavenumber and a particular vertical structure.

\section{Linear theory of leaky trapped modes}

Neglecting the Coriolis force, the vertical velocity perturbations $w$ in the linearized inviscid Boussinesq equations governing steady two-dimensional $(x, z)$ mountain waves satisfy [see Gill 1982, his (8.9.8)]

$$
\left(\frac{\partial^{2}}{\partial x^{2}}+\frac{\partial^{2}}{\partial z^{2}}\right) w+\left(\frac{N^{2}}{U^{2}}-\frac{1}{U} \frac{d^{2} U}{d z^{2}}\right) w=0
$$

where $U(z)>0$ is the basic-state cross-mountain flow and $N(z)$ is the Brunt-Väisälä frequency

$$
N^{2}=\frac{g}{\theta_{0}} \frac{d \bar{\theta}}{d z}
$$

here, $\bar{\theta}(x)$ is the potential temperature in the basic state, $\theta_{0}$ is a constant reference value of the potential temperature, and $g$ is the gravitational acceleration. Assuming a solution of the form $w(x, z)=\hat{w}(z) e^{i k x}$, (1) becomes

$$
\frac{d^{2} \hat{w}}{d z^{2}}+\left(l^{2}-k^{2}\right) \hat{w}=0,
$$

where

$$
l^{2}(z)=\frac{N^{2}}{U^{2}}-\frac{1}{U} \frac{d^{2} U}{d z^{2}}
$$

is the Scorer parameter.

Scorer (1949) obtained solutions to (3) for the specific case of two-layer atmospheres in which $l$ is constant in each layer and the waves are completely trapped in the upper layer. In the following, we obtain eigenfunctions $\hat{w}(z)$ and eigenvalues $k$ for (3) in atmospheres having multiple layers with constant wind shear and static stability in each layer using the numerical procedure described in the appendix. In all cases, the top layer is one with constant $N$ and $U$, implying that $l$ is also constant in that layer. Before discussing the solutions throughout their full vertical extent, let us consider the wave structures in the constant $-N$ and $-U$ upper layer associated with perfectly trapped and leaky modes.

When $N$ and $U$ are constant, solutions to (3) have the form $\hat{w}(z)=e^{i m z}$, where

$$
m= \pm\left(l^{2}-k^{2}\right)^{1 / 2} .
$$

In the case of classical trapped waves with no downstream decay, $k$ is real, $k^{2}>l^{2}$, and the positive root in (5) gives the physically relevant ${ }^{1}$ solution to (1) that decays as $z \rightarrow \infty$,

$$
w(x, z)=e^{-\left[\left(k^{2}-l^{2}\right)^{1 / 2} z\right]} e^{i k x},
$$

where it is understood we take the real part of the solution after multiplying by a complex coefficient determining the phase and amplitude. On the other hand, if $k^{2}<l^{2}$, the classical solution to (1) is a vertically propagating wave of the form

$$
w(x, z)=e^{i(k x+m z)} .
$$

Assuming without loss of generality that $k>0$, the positive root is again taken in (5) to ensure upward energy

\footnotetext{
${ }^{1}$ Recall that we have made the Boussinesq approximation, thereby neglecting the decrease in mean atmospheric density with height.
} 


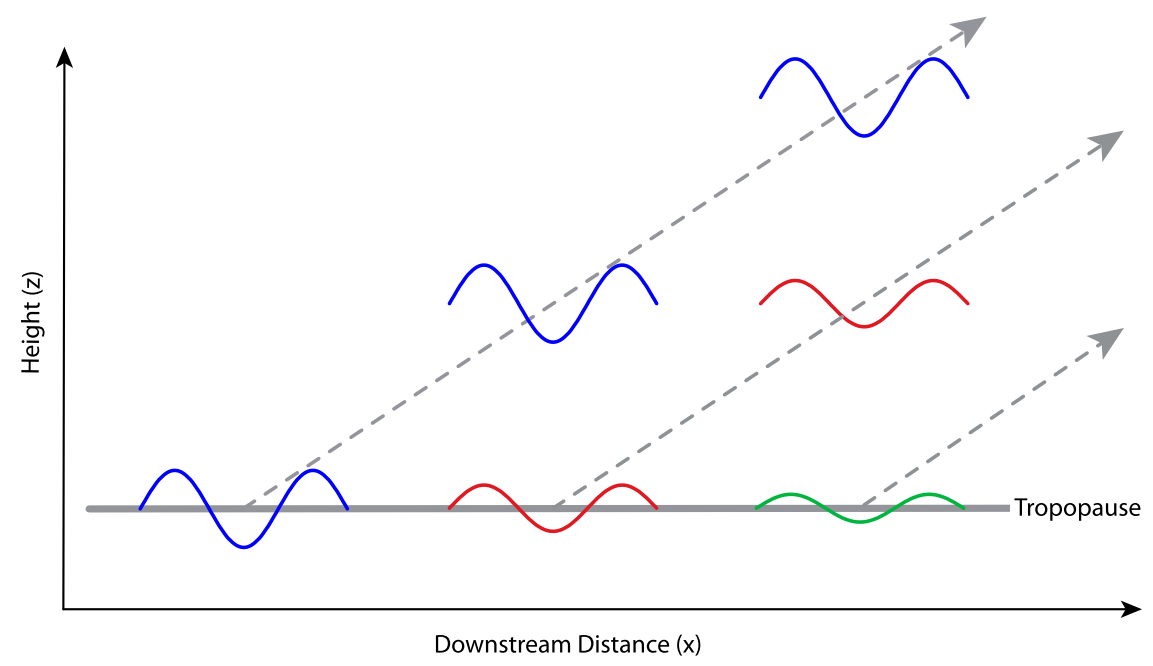

FIG. 1. Schematic illustration of why wave amplitude increases with height at a fixed downstream distance due to the conservative propagation of wave packets through the stratosphere above a train of lee waves that decay downstream. Different packets are shown as they enter the stratosphere progressively farther downstream of the wave source and propagate along ray paths (dashed gray lines with arrowheads) following the group velocity. Blue packets are launched closest to the source; green packets are launched farthest downstream.

propagation. This follows from the expression for the vertical group velocity. The dispersion relation for gravity waves that can be steady (i.e., have a frequency $\omega=0$ ) in a mean flow $U>0$ is

$$
\omega=U k-\frac{N k}{\left(k^{2}+m^{2}\right)^{1 / 2}}
$$

a)

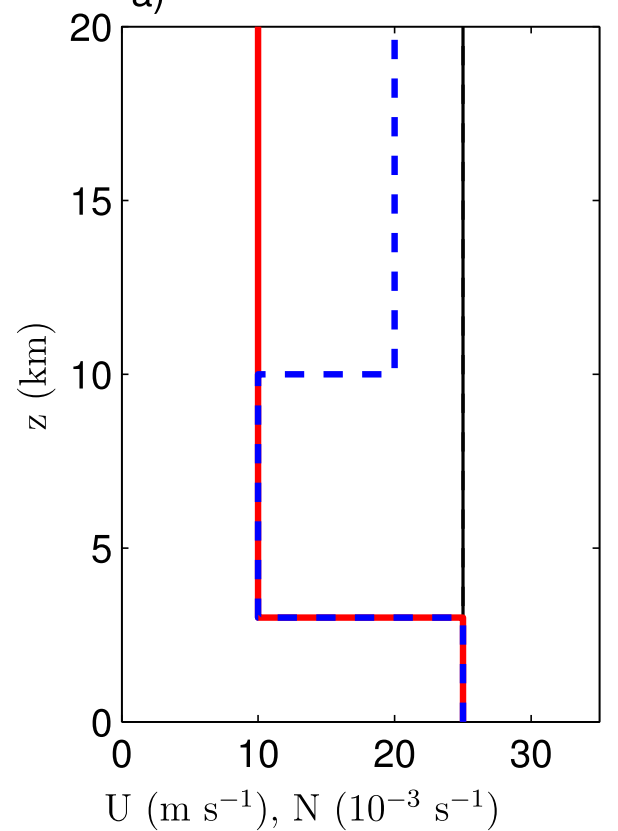

b) No Stratosphere

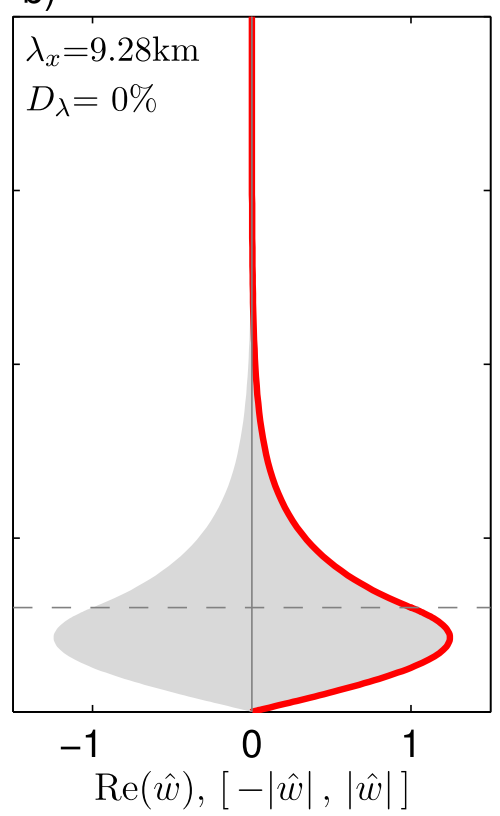

c) $\quad \mathrm{N}_{s}=0.02 \mathrm{~s}^{-1}$

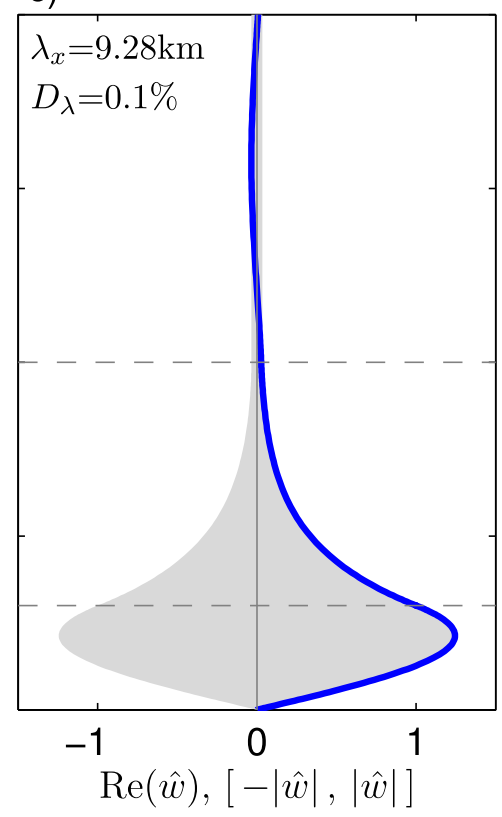

FIG. 2. Basic-state environment and modal structure for profile 1 as a function of height. (a) $U$ (black line) and $N$ (solid red line for no stratosphere, dashed blue for stratosphere); (b) $\operatorname{Re}(\hat{w})($ red line) and the interval $[-|\hat{w}|,|\hat{w}|]$ (shading) for the no-stratosphere case; (c) as in (b), but for the stratosphere case with $\operatorname{Re}(\hat{w})$ in blue. Horizontal wavelength $\lambda_{x}$ and downstream dissipation per wavelength $D_{\lambda}$ are also noted in (b) and (c). 

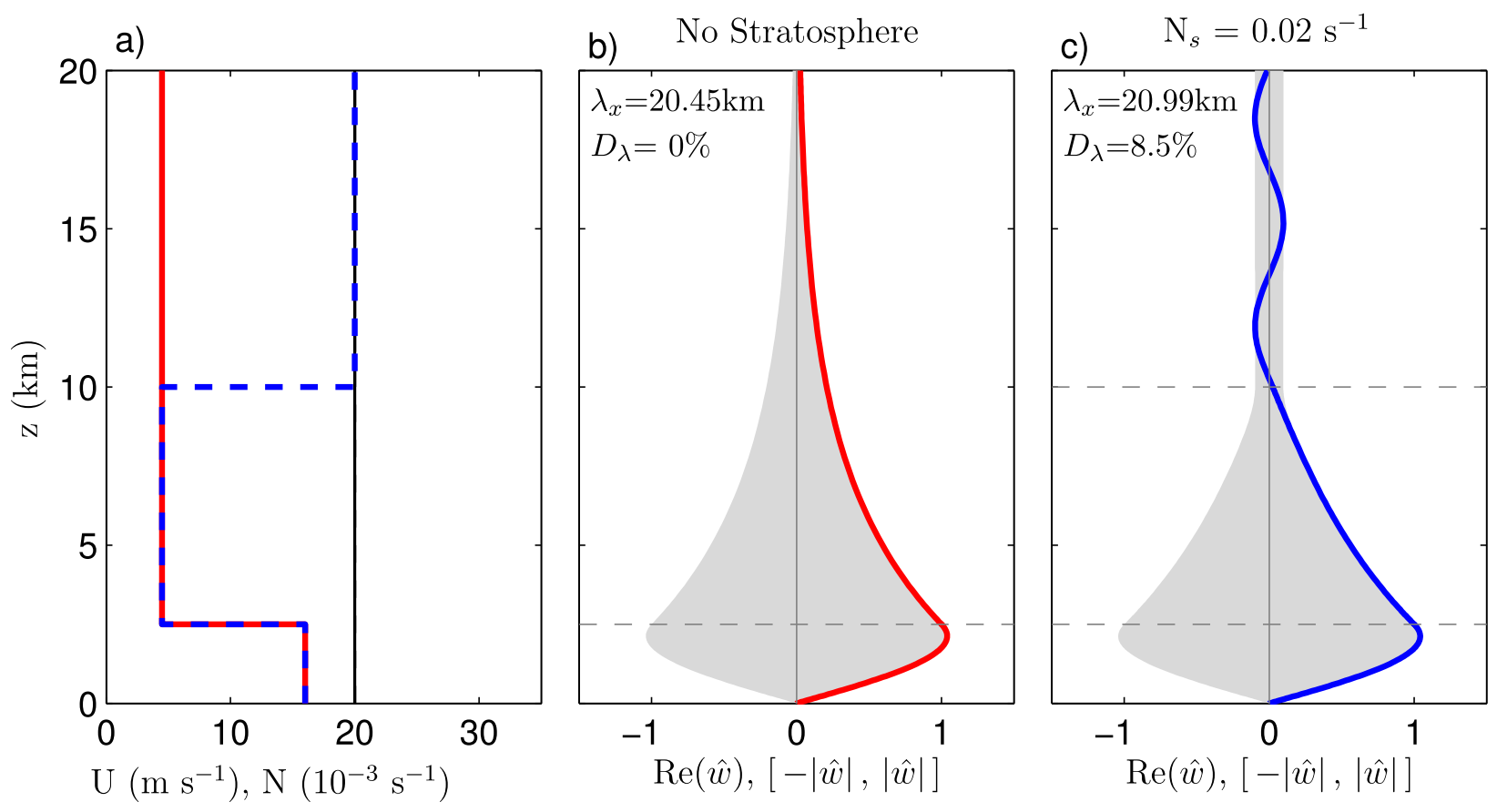

FIG. 3. As in Fig. 2, but for profile 2.

$$
c_{g_{z}} \equiv \frac{\partial \omega}{\partial m}=\frac{N k m}{\left(k^{2}+m^{2}\right)^{3 / 2}}
$$

which is positive when $k$ and $m$ have the same sign.

Since the waves transport energy upward, the purely oscillatory solution (7) cannot describe the disturbance above trapped waves generated by an isolated mountain because upward energy leakage would cause such a mode to decay downstream of the source. To accommodate possible downstream decay, we generalize the preceding by letting $k=k_{r}+i k_{i}$ and $m=m_{r}+i m_{i}$ be complex (with $k_{r}, k_{i}, m_{r}, m_{i}$ all real). The general solution to (1) now has the form

$$
w(x, z)=e^{-k_{i} x-m_{i} z} e^{i\left(k_{r} x+m_{r} z\right)},
$$

where from the steady-state dispersion relation (5),

$$
m^{2}=l^{2}-k_{r}^{2}+k_{i}^{2}-2 i k_{i} k_{r}=A e^{i \phi},
$$

with

$$
\begin{aligned}
& A=\left[\left(l^{2}-k_{r}^{2}+k_{i}^{2}\right)^{2}+\left(2 k_{i} k_{r}\right)^{2}\right]^{1 / 2} \text { and } \\
& \phi=\arctan \left(\frac{-2 k_{i} k_{r}}{l^{2}-k_{r}^{2}+k_{i}^{2}}\right) .
\end{aligned}
$$

Without loss of generality, we assume $k_{r}>0$ and choose the sign of $m_{r}$ to match that of $k_{r}$ to give upward energy transport. If the wave decays downstream, $k_{i}>0$. Using the positivity of $k_{r}, k_{i}$, and $m_{r}$, and noting that $l^{2}>k_{r}^{2}$ because the wave is propagating vertically, (13) implies that both $\phi$ and $\phi / 2$ lie in the interval $[-\pi / 2,0]$, and thus $m_{i}<0$. As a consequence, at any fixed value of $x$, the amplitude of the solution (10) grows without bound as $z \rightarrow \infty$.

Unbounded growth in the wave amplitude with height is nonphysical when the wave structure is purely sinusoidal in $x$ and exponential in $z$, which led us to the choice of the decaying solution in (6). Is the solution (10) also unphysical, or can it describe the true stratospheric signal in a wave train downstream from a localized source? We are aware of only one previous publication in which exponential growth with height has been connected with the downstream decay of trapped lee waves: ${ }^{2}$ Tannhauser and Moiseyev (1995) briefly note that in their leaky-wave solutions "...the amplitude diverges $^{3}$ as $z \rightarrow \infty$, this divergence has no clear physical meaning."

Here we present the physical explanation for the increase in amplitude with height. First note that this

\footnotetext{
${ }^{2}$ Figures 5 and 6 of Keller (1994) show decaying trapped waves increasing in amplitude with height in the stratosphere in a linear Boussinesq solution, but this behavior is not mentioned in the text.

3 “Amplitude diverges" presumably refers to exponential growth with height.
} 
increase in amplitude with height is not due to the decrease in atmospheric density with height because we have used the Boussinesq equations to describe the waves. The explanation is easily obtained under the assumption that the downstream decay over an individual trapped wavelength is small, so that one can apply the concepts of group velocity and ray-tracing theory in a WKB sense to gravity waves propagating through the constant $-N$ and $-U$ stratosphere. From (8), the $x$ component of the group velocity is

$$
c_{g_{x}} \equiv \frac{\partial \omega}{\partial k}=U-\frac{N m^{2}}{\left(k^{2}+m^{2}\right)^{3 / 2}} .
$$

In steady waves, (8) reduces to $l^{2}=k^{2}+m^{2}$, and for such waves (14) and (9) simplify to

$$
c_{g_{x}}=U \frac{k^{2}}{l^{2}}, \quad c_{g_{z}}=U \frac{k m}{l^{2}},
$$

and thus

$$
\frac{c_{g_{z}}}{c_{g_{x}}}=\frac{m}{k},
$$

which is positive for waves propagating energy upward.

Since $N$ and $U$ are constant in the stratosphere, conservation of wave action reduces to conservation of wave amplitude, and each wave packet entering the stratosphere will propagate upward and downstream along a ray path parallel to the group velocity vector as schematically illustrated in Fig. 1 . Those packets that enter the stratosphere closest to the wave source have larger amplitude and follow a ray path that places them above packets of lower-amplitude waves entering the stratosphere farther downstream. Thus, at a given value of $x$, the wave amplitude will increase exponentially with height-except that if the leaky trapped wave train is generated by a compact source, the wave amplitude ultimately drops to zero above some threshold value $z_{t}(x)$.

Suppose the leaky trapped waves are generated by a compact tropospheric source at $x_{s}$ where the tropopause height is $h_{s}$, then this threshold may be approximated as

$$
z_{t}(x)=h_{s}+\frac{m}{k}\left(x-x_{s}\right) .
$$

Assuming the compact source does not directly generate a disturbance in the stratosphere, there will be no disturbance above $z_{t}(x)$ because all ray paths in this region originate upstream of the source. In the same manner that we ignore the way the wave amplitude in (10) grows exponentially upstream of a compact source as $x \rightarrow-\infty$, we should ignore the exponential growth as $z \rightarrow \infty$ above $x_{0}$ for all values of $z$ higher than about $z_{t}\left(x_{0}\right)$.
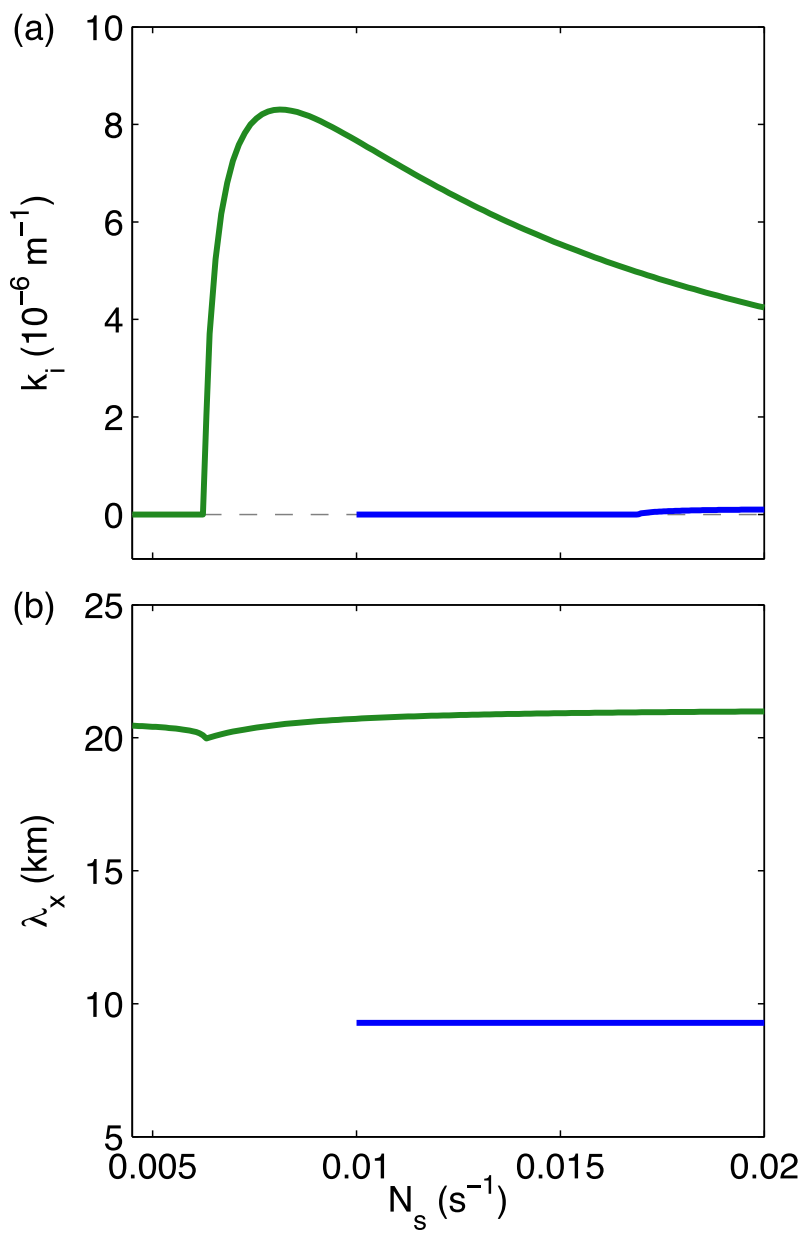

FIG. 4. (a) Rate of downstream decay $k_{i}$ and (b) resonant wavelength $\lambda_{x}$ as a function of stratospheric stability for profiles 1 (blue) and 2 (green).

\section{Three-layer atmospheres with constant wind speed}

As a starting point, we solve the eigenvalue problem (3) for a pair of simple two-layer atmospheres that support trapped waves and examine how those solutions change when a stratosphere is added as a third layer. In these cases, $N$ is constant within each layer and $U$ is constant throughout the entire depth.

One trapped-wave profile (herein profile 1) that has been used in previous studies (Doyle and Durran 2002; Jiang et al. 2006) is a 3-km-deep lower layer in which $N_{l}=0.025 \mathrm{~s}^{-1}$, with $N_{u}=0.01 \mathrm{~s}^{-1}$ above and $U=25 \mathrm{~m} \mathrm{~s}^{-1}$ at all levels. The effect of adding a stratosphere to this profile is illustrated in Fig. 2. As plotted in Fig. 2a, the stratospheric stability is $N_{s}=0.02 \mathrm{~s}^{-1}$ and the tropopause height $h_{s}$ is $10 \mathrm{~km}$. Comparing the vertical structure of the purely trapped two-layer solution (Fig. 2b) with that in the presence of the stratosphere (Fig. 2c), it 

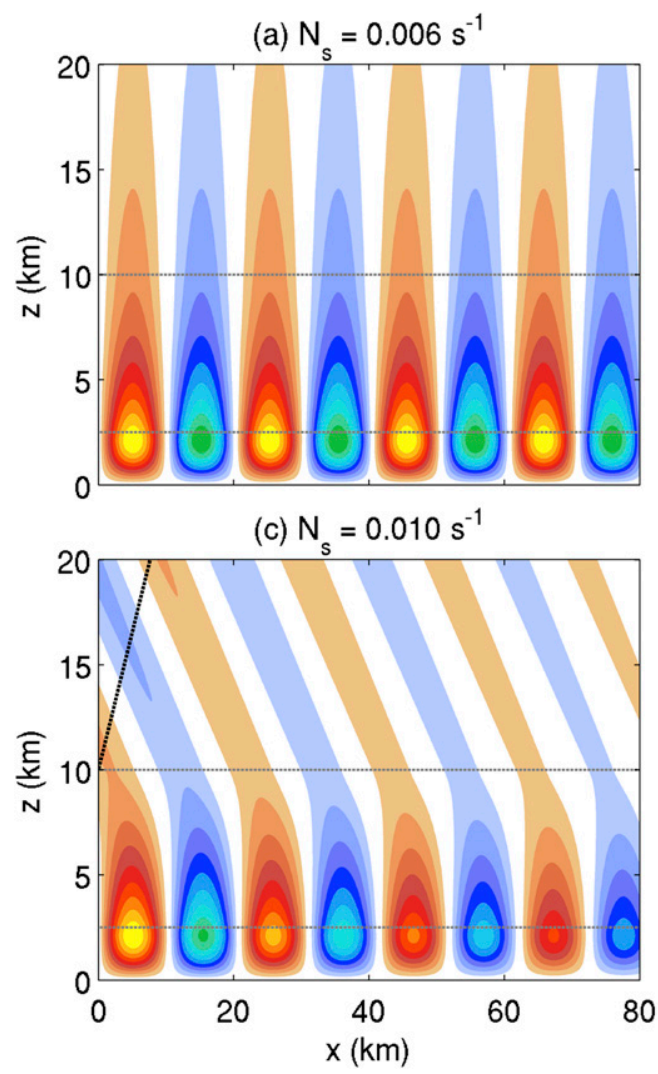

(b) $\mathrm{N}_{\mathrm{s}}=0.008 \mathrm{~s}^{-1}$

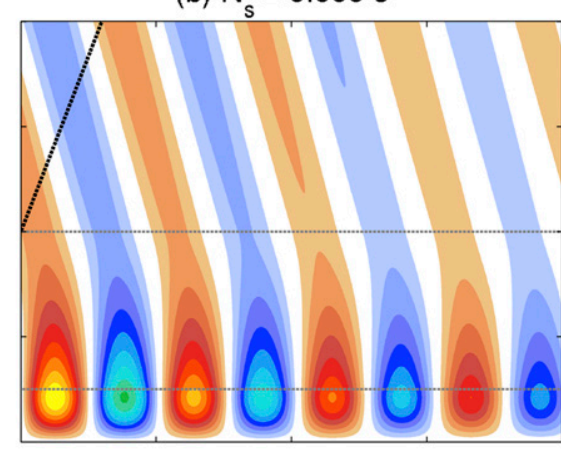

(d) $\mathrm{N}_{\mathrm{s}}=0.020 \mathrm{~s}^{-1}$

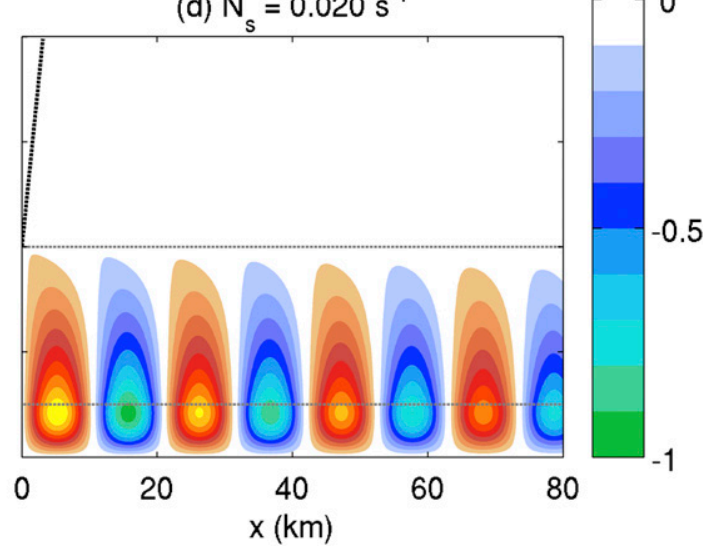

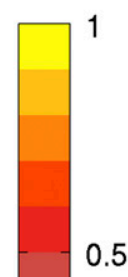

0 .5

FIG. 5. Contours of $w$ in a vertical cross section for modes supported by profile 2 with $h_{s}=10 \mathrm{~km}$ and $N_{s}$ equal to (a) 0.006 , (b) 0.008 , (c) 0.010 , and (d) $0.020 \mathrm{~s}^{-1}$. In all cases, the waves are plotted for a flow from left to right and the maximum $w$ is normalized to unity in the first updraft. Contours for $w$ are at intervals of 0.1. Dashed gray lines indicate the top of the lowest layer and the tropopause; the thin dashed black lines in (b)-(d) are parallel to the group velocity vector.

is clear that the mode is insensitive to the stratosphere, which is consistent with the conclusions of Corby and Sawyer (1958).

Ralph et al. (1997) surveyed observations of 26 leewave events collected by several different researchers. They found the average wavelength during these events was $15.8 \pm 4.5 \mathrm{~km}$. The shortest average wavelength was $8.3 \mathrm{~km}$; the longest was $28.6 \mathrm{~km}$. The resonant wavelength supported by profile 1 is $9.3 \mathrm{~km}$-shorter than all but one of the events surveyed in Ralph et al. (1997). Figure 3 shows how the results change if we add the same stratosphere $\left(N_{s}=0.02 \mathrm{~s}^{-1}, h_{s}=10 \mathrm{~km}\right)$ to another simple profile (profile 2) that supports a two-layer solution with a 20.5-km resonant wavelength. In this case $N_{l}=0.016 \mathrm{~s}^{-1}$ throughout a $2.5-\mathrm{km}$-deep layer, $N_{u}=0.0045 \mathrm{~s}^{-1}$ and $U=$ $20 \mathrm{~m} \mathrm{~s}^{-1}$ (Fig. 3a). When the stratosphere is present, the resonant horizontal wavelength increases slightly to $21.0 \mathrm{~km}$, but more significantly, vertical propagation carries a nontrivial fraction of the wave amplitude upward throughout the stratosphere. This upward propagation is evident in the pronounced oscillatory structure of $\operatorname{Re}(\hat{w})$ and in the width of the interval $[-|\hat{w}|,|\hat{w}|]$ (indicated by the shading), which does not decrease with height in the stratosphere.

The downstream decay rate $k_{i}$ and the wavelength $\lambda_{x}=2 \pi / k_{r}$ are plotted as a function of stratospheric stability in Fig. 4 for both profiles 1 and 2; in these cases $h_{s}$ is held fixed at $10 \mathrm{~km}$. Vertical propagation into the stratosphere occurs when $N_{s}>U k_{r}$, which evaluates to about $N_{s}>0.017 \mathrm{~s}^{-1}$ for profile 1 and $N_{s}>0.0063 \mathrm{~s}^{-1}$ for profile 2. Downstream decay occurs very slowly for profile 1 but is almost two orders of magnitude greater for profile 2 and rises rapidly to a maximum soon after $N_{s}$ exceeds the threshold for decay. The decay rate then gradually asymptotically approaches zero, which is the value for a rigid lid in the limit $N_{s} \rightarrow \infty$. The horizontal wavelength is only slightly sensitive to $N_{s}$ (Fig. $4 \mathrm{~b}$ ), with most of the variation occurring in profile 2 near the threshold for vertical propagation.

Vertical cross sections of $w$ for four profile-2 modes with different stratospheric stabilities are plotted in Fig. 5. When $N_{s}=0.006 \mathrm{~s}^{-1}$ (Fig. 5a), the waves do not 
(a) Mesoscale model

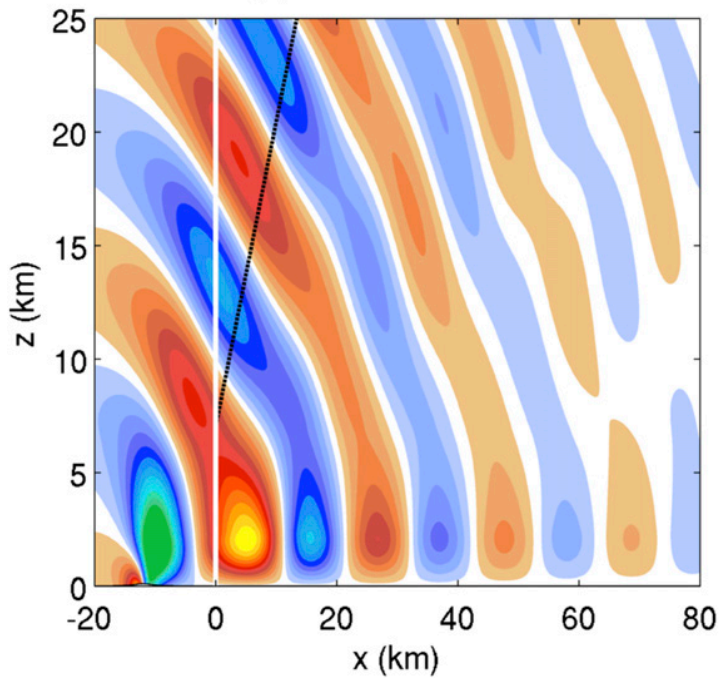

(b): Linear Model

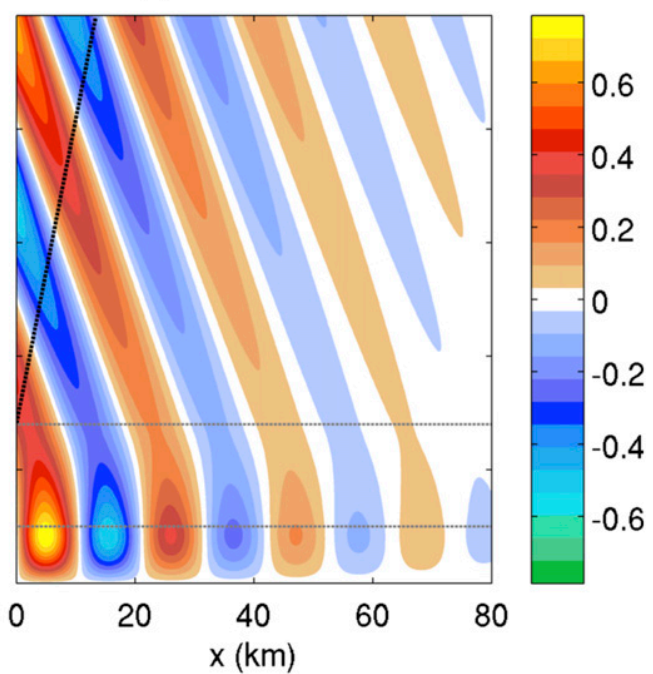

FIG. 6. Contours of $w$ in a vertical cross section for profile 2 with $N_{s}=0.01 \mathrm{~s}^{-1}$ and $h_{s}=7 \mathrm{~km}$ from (a) the steadystate limit approached by the time-dependent numerical solution and (b) the linear model. Units for $w$ are meters per second, and $w$ from the linear model is normalized so its maximum value matches that for the numerical solution in the first updraft. The thin dashed black lines are parallel to the group velocity vector. The $100-\mathrm{m}$-high mountain is barely visible at about $x=-10 \mathrm{~km}$ in (a). The vertical white line in (a) separates the near-mountain solution from the downstream portion, which may be compared with the linear mode in (b).

propagate vertically in the stratosphere, and there is no loss of amplitude downstream. As $N_{s}$ is increased to $0.008 \mathrm{~s}^{-1}$, the downstream decay rate is near its maximum (as is the growth with height at a fixed value of $x$ ). Further increases in $N_{s}$ do not lead to stronger downstream decay, and as suggested by Fig. 4a, the downstream decay is noticeably weaker when $N_{s}=0.02 \mathrm{~s}^{-1}$ (Fig. 5d). As predicted by theory, the vertically propagating waves in the stratosphere develop an upstream phase tilt that increases as the strength of $N_{s}$ is increased.

Also plotted in Figs. 5b-d is a dashed line emanating from $(x, z)=(0,10) \mathrm{km}$ parallel to the group velocity vector. As particularly evident in Fig. 5c, the wave amplitude in the stratosphere is constant along this line. If the waves were generated by a compact source at $x=0$ that did not extend into the stratosphere, this dashed line would represent the threshold $z_{t}(x)$ defined in (16), above which the atmosphere would be unperturbed. The actual disturbance generated by an isolated mountain will, however, include longer-wavelength vertically propagating waves, and the signal from such waves can produce oscillations in the region above the dashed lines in Figs. 5b-d.

Figure 6 compares the vertical velocities in a numerical solution for the full set of waves generated by a Witch of Agnesi mountain with a half-width of $2.5 \mathrm{~km}$ to those for the leaky-mode linear solution. The atmospheric structure is given by profile 2 with $N_{s}=0.01 \mathrm{~s}^{-1}$ and a tropopause height of $7 \mathrm{~km}$. The numerical solution is from the time-dependent mountain wave model discussed in Part II. An effectively linear solution is obtained from the nonlinear numerical model by using a mountain just $100 \mathrm{~m}$ high; the solution has become completely steady by the time shown in Fig. 6a. The amplitude of the linear solution in Fig. $6 \mathrm{~b}$ has been scaled to match that of the first updraft downstream of the mountain in the numerical solution. ${ }^{4}$ The agreement between the numerical solution and the linear mode in the region below the dashed black lines is very good, with almost identical resonant wavelengths, downstream decay, and growth with height at a fixed value of $x$. Additional modes triggered by the mountain are visible above and slightly downstream of the mountain in Fig. 6a, and some of these waves directly excite the leaky trapped mode in the stratosphere accounting for much of the signal above the dashed line in the numerical simulation.

When the tropopause is at $10 \mathrm{~km}$, the trapped wave supported by profile 1 decays almost completely before it encounters the stratosphere, whereas that supported by profile 2 retains approximately $20 \%$ of its maximum

\footnotetext{
${ }^{4}$ Observations (Smith 1976) and numerical simulations (Nance and Durran 1997) have shown that the lee-wave amplitude in typical atmospheric flows is often significantly underestimated by linear theory, even when other aspects of the wave structure are in good agreement. We therefore do not attempt to directly calculate the amplitude of linear leaky waves forced by a given topography.
} 

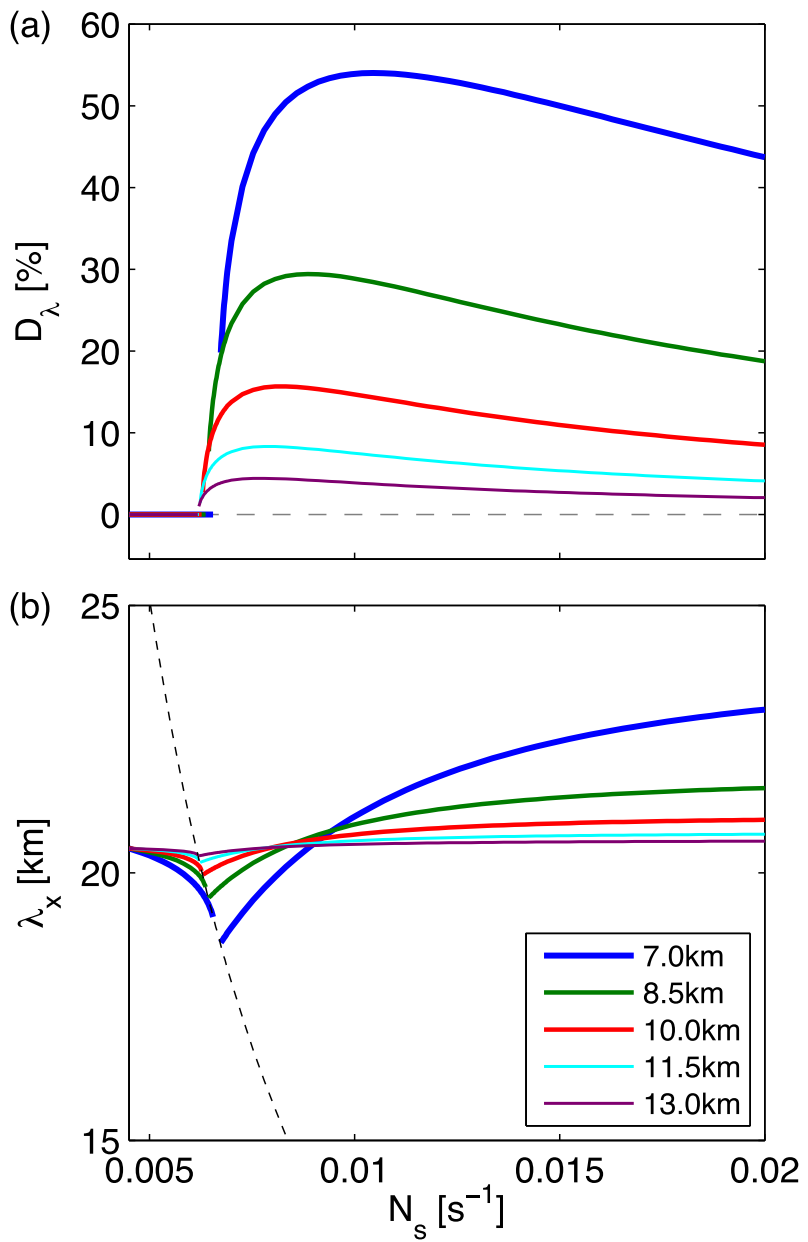

FIG. 7. Influence of $h_{s}$ and $N_{s}$ in profile 2 on (a) downstream damping per unit wavelength $D_{\lambda}$ and (b) resonant wavelength $\lambda_{x}$. Individual curves show the values for tropopause heights ranging between 7 and $13 \mathrm{~km}$. The dashed line in (b) shows the curve along which $l_{s}=2 \pi / \lambda_{x}$.

amplitude at the tropopause level (cf. Figs. $2 \mathrm{~b}$ and $3 \mathrm{~b}$ ). The slower decay of the profile- 2 mode through the upper troposphere facilitates the leakage of energy into the stratosphere, so not surprisingly, the downstream decay of the profile- 2 modes is increased if the height of the tropopause is lowered. This is shown in Fig. 7a, in which the downstream damping per unit horizontal wavelength $D_{\lambda}$ is plotted as a function of $N_{s}$ for several different $h_{s}$; the decay of profile- 2 modes can exceed $50 \%$ per wavelength when the tropopause is lowered to $7 \mathrm{~km}$ (the elevation for the case shown in Fig. 6). Let $N_{s}^{*}$ denote the value of $N_{s}$ that produces the maximum decay for a given tropopause height. For the heights shown in Fig. 7, and for values of $N_{s}$ greater than $N_{s}^{*}, D_{\lambda}$ is much more sensitive to the tropopause height than to stratospheric stability. On the other hand, $D_{\lambda}$ is very sensitive to $N_{s}$ for values between the threshold for leakage and $N_{s}^{*}$.
In contrast to the downstream decay, the resonant wavelength is only moderately sensitive to the height of the tropopause when $h_{s}$ is relatively low (at 7 or $8 \mathrm{~km}$ ) and is quite insensitive when it is higher (Fig. 7b). Also plotted as the dashed black line in Fig. 7b is the curve along which the Scorer parameter in the stratosphere $l_{s}$ equals the horizontal wavenumber. The right-hand side of (5) is zero along this curve, marking the transition from solutions that decay exponentially with height to solutions that contain a vertically propagating wave component. The behavior at $l_{s}=k$ is singular, and our method fails to converge in a small neighborhood around this line. Gaps appear in our curves at those points. ${ }^{5}$

\section{Elevated inversion and tropospheric wind shear}

As demonstrated in the preceding section, the rate at which trapped lee waves dissipate downstream owing to stratospheric leakage can vary dramatically depending on the specifics of the atmospheric profile. Neither profiles 1 nor 2 are good approximations to the atmospheric structure during actual lee-wave events. Instead of a very stable layer extending 2.5 or $3 \mathrm{~km}$ up from the surface, observations often show a weakly stratified (or neutral) layer at the surface capped at an elevation of $2-4 \mathrm{~km}$ by a strongly stable layer whose thickness seldom exceeds $1 \mathrm{~km}$ and is sometimes no thicker than $250 \mathrm{~m}$. Moreover, in contrast to the unsheared upstream winds in profiles 1 and 2, real-world trapped lee waves are usually associated with large increases with height in the cross-mountain wind component that significantly reduce the value of $N / U$ and thereby promote trapping. To assess the actual importance of stratospheric leakage in typical atmospheric cases we must look at more realistic profiles.

Profile 3 is more representative of actual trappedwave events. As indicated by the plots of $U(z)$ and $N(z)$ in Fig. 8a, the cross-mountain wind speed increases from 10 to $35 \mathrm{~m} \mathrm{~s}^{-1}$ across the troposphere and remains constant in the stratosphere; in addition, $N=0.003 \mathrm{~s}^{-1}$ between the surface and $3 \mathrm{~km}, N=0.025 \mathrm{~s}^{-1}$ in an inversion between 3.0 and $3.3 \mathrm{~km}$, and $N=0.01 \mathrm{~s}^{-1}$ through the remainder of the troposphere. The influence of tropopause height on two leaky profile- 3 modes is compared for the case $N_{s}=0.02 \mathrm{~s}^{-1}$ in Figs. 8b,c. As $h_{s}$ increases from 8.5 to $10 \mathrm{~km}$, the resonant wavelength

\footnotetext{
${ }^{5} \mathrm{We}$ also do not plot converged but untrapped solutions for $k_{r}<l_{s}$, partly for clarity and partly because some of these were very strongly damped and appeared to be on a different branch of solutions.
} 
a)

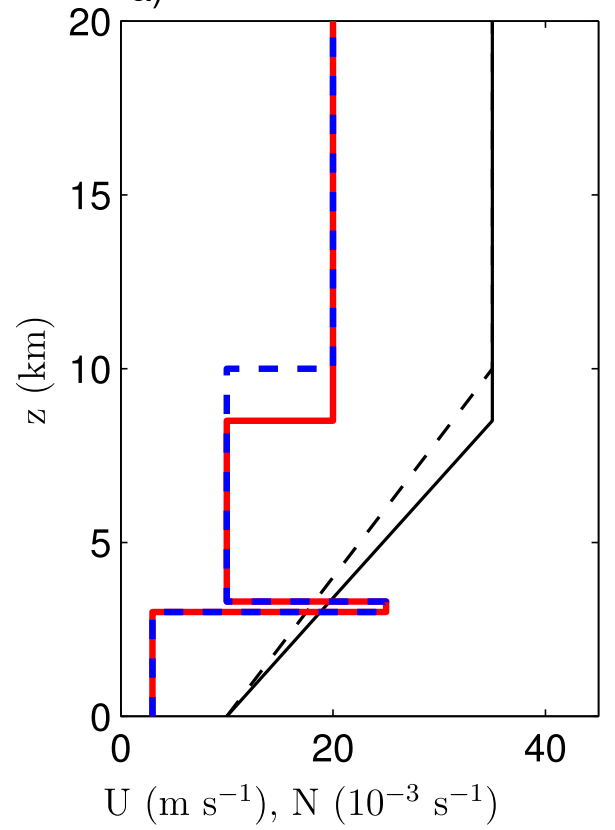

b) $\mathrm{h}_{s}=8.5 \mathrm{~km}$

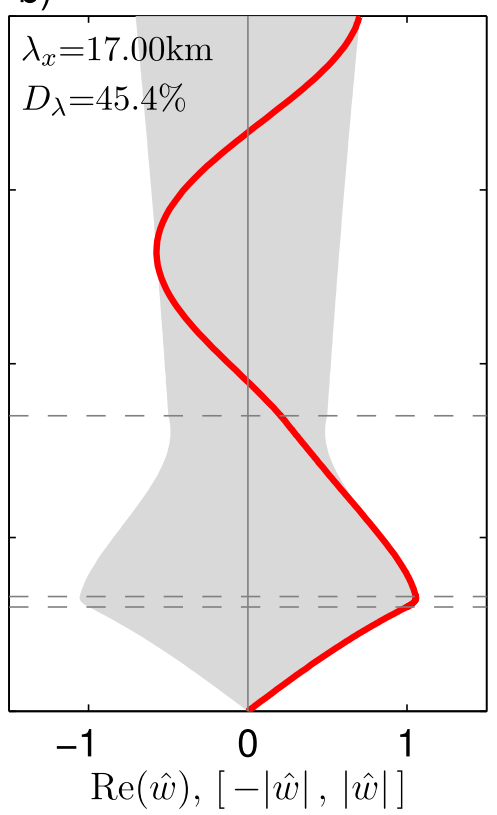

c) $\mathrm{h}_{\mathrm{s}}=10.0 \mathrm{~km}$

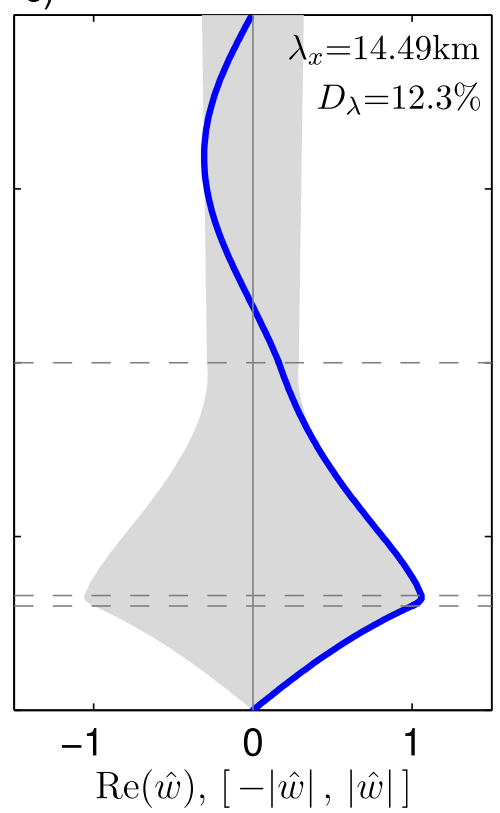

FIG. 8. As in Fig. 2 for profile 3, except both cases have a stratosphere with $N_{s}=0.02 \mathrm{~s}^{-1}$ at a height of (b) 8.5 and (c) $10 \mathrm{~km}$. The dashed lines in (a) plot properties for the 10-km case; the thin dashed lines in (b) and (c) indicate the locations of the inversion and the tropopause.

decreases from 17.0 to $14.5 \mathrm{~km}$ and the fraction of the wave amplitude entering the stratosphere is almost halved.

Cross sections of $w$ are plotted in Fig. 9 for four profile-3 modes with different combinations of $N_{s}$ and $h_{s}$. When there is no increase in static stability above the troposphere (Fig. 9a), the waves are completely trapped and $\lambda_{x}=16.4 \mathrm{~km}$. The remaining panels show the influence of tropopause height when $N_{s}=0.02 \mathrm{~s}^{-1}$. In Fig. $9 \mathrm{~b}, h_{s}=8.5 \mathrm{~km}$ (the same case as in Fig. $8 \mathrm{~b}$ ) and the solution decays rapidly downstream. Coupled with this rapid downstream decay is a strong increase in the wave amplitude with height at a constant value of $x$. Increasing $h_{s}$ to $9 \mathrm{~km}$ (Fig. 9c) reduces the rate of downstream decay, but even in this case the strength of the updraft feeding the third lee wave is reduced by more than $50 \%$, which, if appropriate humidity values were present, could easily be enough to prevent visible wavecloud formation. Not surprisingly, increasing $h_{s}$ to $10 \mathrm{~km}$ (Fig. 9d, also the case in Fig. 8c) further reduces the downstream decay.

The downstream damping per wavelength for profile3 modes is plotted as a function of $N_{s}$ in Fig. 10a for several values of $h_{s}$. The curves for $D_{\lambda}$ are similar to those for profile 2 in Fig. 7a, although the numerical values are considerably different. Here the value of $N_{s}$ for which leaky modes decay most rapidly is much closer to the typical lower-stratospheric stability of $N_{s}=$ $0.02 \mathrm{~s}^{-1}$ and the decay rates are larger (cf. the values for $h_{s}=8.5 \mathrm{~km}$ ). Figure $10 \mathrm{~b}$ shows the influence of $N_{s}$ and $h_{s}$ on the horizontal wavelength. The shortest $\lambda_{x}$ occur near the value for which $l_{s}=k$ (indicated by the dashed line), which is similar to the behavior for profile 2 (Fig. $5 b$ ); but, in contrast to the situation for profile 2 , there is also a clear tendency for increases in $h_{s}$ to decrease $\lambda_{x}$ over the full range of $N_{s}$.

Because the elevated stable layer plays a key role in supporting actual trapped lee waves, let us consider the sensitivity of the solution to changes in the thickness of this layer in the context of the overall profile- 3 sounding with $N_{s}=0.02 \mathrm{~s}^{-1}$ and $h_{s}=10 \mathrm{~km}$. The elevation of the bottom of the stable layer is held constant at $3.0 \mathrm{~km}$. First consider thickness changes in which the static stability within the inversion is held constant. As shown by the blue curves in Fig. 11, as the layer thickness increases both $D_{\lambda}$ and $\lambda_{x}$ decrease. If the layer becomes thicker than about $550 \mathrm{~m}$, the wave becomes completely trapped. In the real world, however, increases in the thickness of an elevated stable layer are often accompanied by reductions in the value of $N$ within the layer. The green lines in Fig. 11 show the influence of changes in stable layer thickness when the total potential temperature change across the layer $\Delta \theta_{i}$ is held constant. In contrast to the case with fixed $N$, as the thickness of the 
(a) No Stratosphere

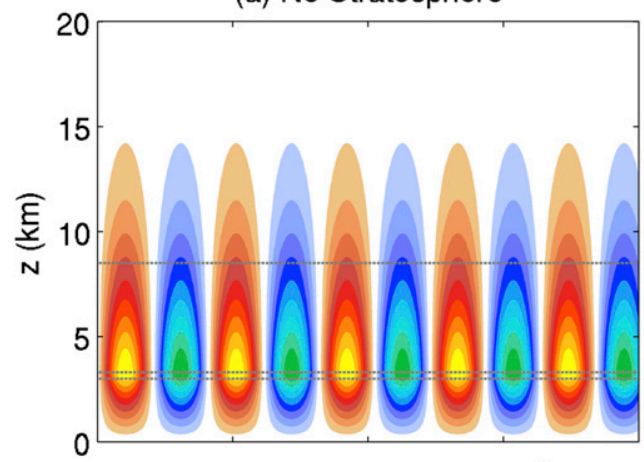

(c) $h_{s}=9 \mathrm{~km}, N_{s}=0.02 \mathrm{~s}^{-1}$

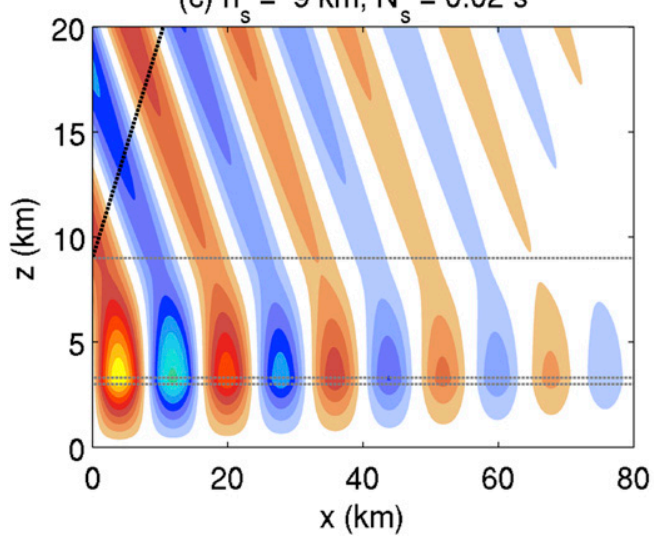

(b) $\mathrm{h}_{\mathrm{s}}=8.5 \mathrm{~km}, \mathrm{~N}_{\mathrm{s}}=0.02 \mathrm{~s}^{-1}$

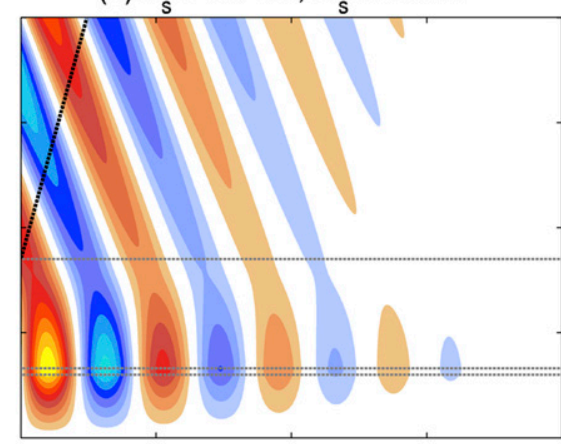

(d) $h_{s}=10 \mathrm{~km}, N_{s}=0.02 \mathrm{~s}^{-1}$

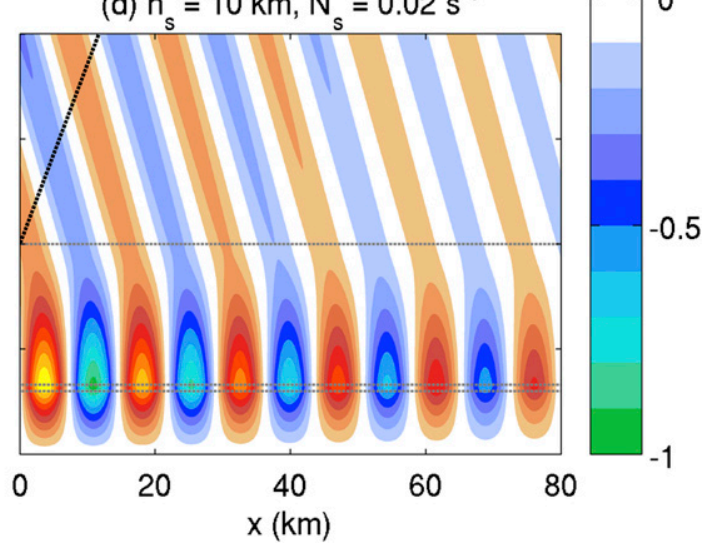

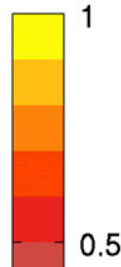

0

.5

FIG. 9. As in Fig. 5, but for profile 3. The tropopause height and static stability are varied such that $N_{s}=($ a) 0.01 and (b)-(d) $0.02 \mathrm{~s}^{-1}$, and $h_{s}=$ (a),(b) 8.5 , (c) 9 , and (d) $10 \mathrm{~km}$. Thin dashed gray lines indicate the top and bottom of the inversion and the tropopause.

constant- $\Delta \theta_{i}$ stable layer increases, both $D_{\lambda}$ and $\lambda_{x}$ increase.

Now consider the influence of changes in the height of the inversion $h_{i}$ (measured between the ground and the top of the layer). Our focus is again on profile 3 , with $N_{s}=0.02 \mathrm{~s}^{-1}$ and $h_{s}=10 \mathrm{~km}$. We examine a pair of inversions having the same $\Delta \theta_{i}$ such that $N=0.025 \mathrm{~s}^{-1}$ in the thinner 300-m-thick inversion. As shown in Fig. 12, both $D_{\lambda}$ and $\lambda_{x}$ increase as the elevation of the inversion rises. Qualitatively similar results are obtained for both thin $(300 \mathrm{~m})$ and thick $(1000 \mathrm{~m})$ inversions, and the variation in the damping over the range $2 \leq h_{i} \leq 6 \mathrm{~km}$ is extreme, ranging from less than $10 \%$ per wavelength at $h_{i}=2 \mathrm{~km}$ to $90 \%$ when $h_{i}=6 \mathrm{~km}$. The horizontal wavelength approximately doubles over the same range of $h_{i}$, from 12 to $25 \mathrm{~km}$ in the $300-\mathrm{m}$-thick case.

\section{Stratospheric wind shear}

Profile 3 differs from actual atmospheric soundings in that the stratospheric winds are constant with height and equal to the maximum tropospheric wind speed. In reality, the stratospheric winds decrease rapidly with height above the jet core. We therefore consider profile 4 , which is identical to the family of profile- 3 soundings except that reverse wind shear is added in the lower stratosphere such that the $35 \mathrm{~m} \mathrm{~s}^{-1}$ jet maximum is maintained through a 1-km-deep layer and then topped by a $2.5-\mathrm{km}$ layer throughout which the wind speed linearly decreases to $10 \mathrm{~m} \mathrm{~s}^{-1}$. The resulting sounding is indicated by the dashed lines in Fig. 13a.

The influence of reversed stratospheric shear on the vertical structure of leaky trapped modes is compared in Figs. 13b,c. The reduction in $U$ to a constant value of $10 \mathrm{~ms}^{-1}$ dramatically reduces the vertical wavelength in the top of the domain. The shear layer also creates partial reflections into downward-propagating modes that can produce complex wave structures such as the secondary extrema in $w$ apparent at $z=10 \mathrm{~km}$ in Fig. 14a. Nevertheless, such pronounced secondary extrema are not typically present. If, for example, the tropopause is raised from 8.0 to $10.0 \mathrm{~km}$ while holding all other parameters constant, the secondary extrema in the shear layer are far less obvious (Fig. 14b).

At least for modes similar to those supported by profiles 3 and 4, the reversed shear above the jet core 

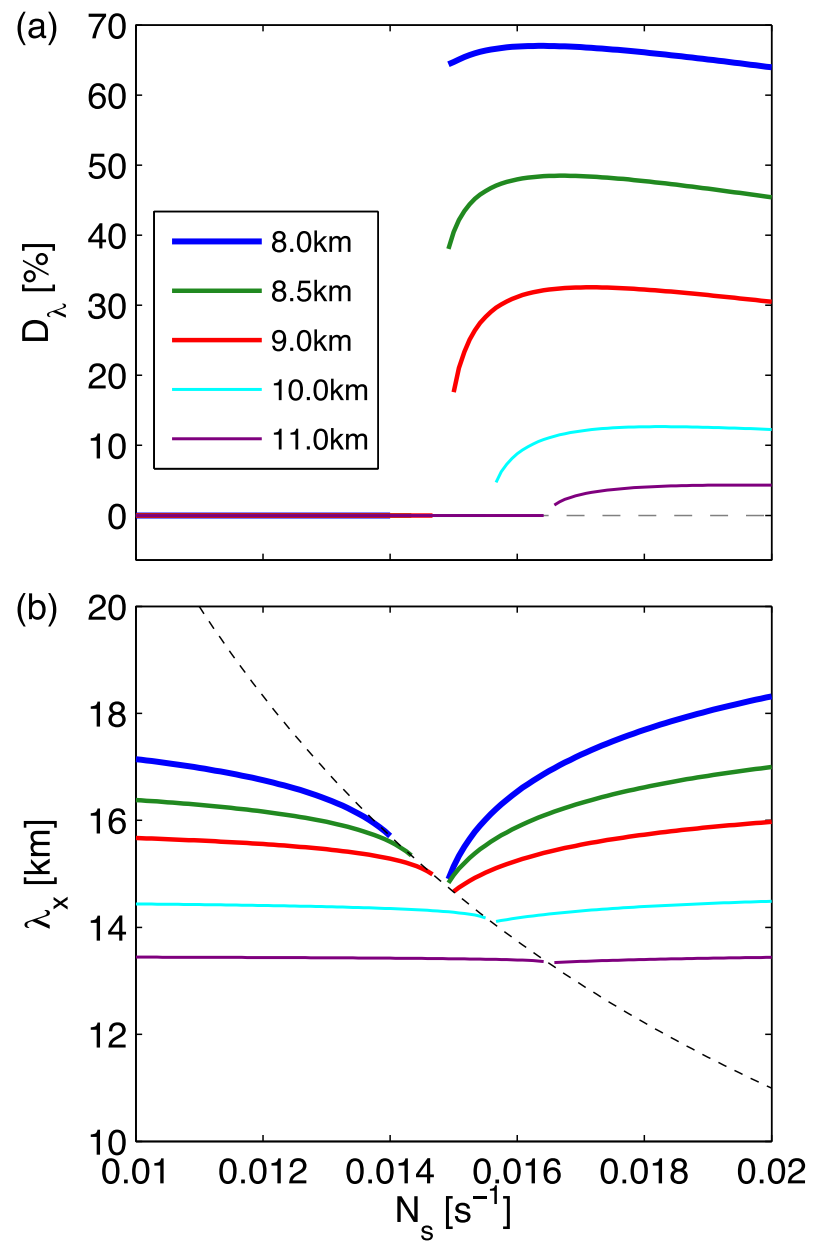

FIG. 10. As in Fig. 7, but for profile 3.

appears to exert only a modest influence on the downstream decay and horizontal wavelength of leaky trapped waves. This is demonstrated in Fig. 15, in which $D_{\lambda}$ and $\lambda_{x}$ are plotted as a function of the height of the tropopause (with the 2.5-km-deep reversed shear layer beginning $1 \mathrm{~km}$ above the tropopause). ${ }^{6}$ When the tropopause is low (near $8 \mathrm{~km}$ ), stratospheric shear increases $\lambda_{x}$ by roughly $5 \%$ while decreasing $D_{\lambda}$ by about $10 \%$, but these differences almost vanish for tropopause heights above $10 \mathrm{~km}$.

\section{Higher-order modes}

None of the modes considered so far include nodal lines, which occur in higher-order trapped modes. In two-layer atmospheres with constant Scorer parameters

\footnotetext{
${ }^{6}$ Recall that $D_{\lambda}$ and $\lambda_{x}$ are much more sensitive to $h_{s}$ than $N_{s}$ (Fig. 10).
}
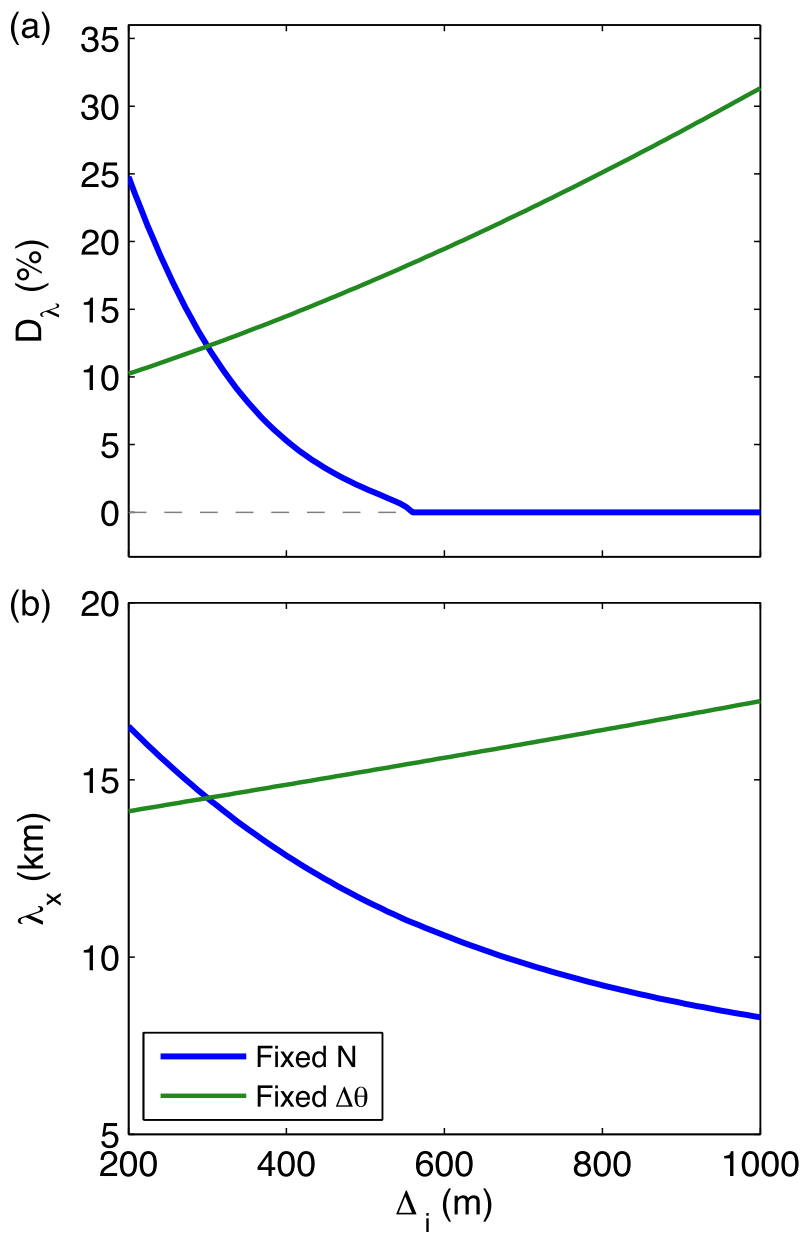

FIG. 11. Influence of the thickness of the elevated stable layer in profile 3 on (a) downstream decay per wavelength and (b) resonant wavelength. Blue curves are for fixed $N$ within the stable layer; green curves are for fixed $\Delta \theta$ across the layer.

$l_{l}$ and $l_{u}$ in the lower and upper layers respectively, the condition for the existence of $M$ trapped modes is

$$
\left[\frac{(2 M+1) \pi}{2 H}\right]^{2} \leq l_{l}^{2}-l_{u}^{2} \leq\left[\frac{(2 M-1) \pi}{2 H}\right]^{2}
$$

where $H$ is the depth of the lower layer (Queney et al. 1960). We are not aware of any definitive atmospheric observations of higher-order lee-wave modes similar to those supported by the two-layer model. This is probably because the atmospheric structures capable of supporting such modes almost never occur. For example, the $M=2$ mode is supported by profile 1 only if the depth of the extremely stable lower layer exceeds $5.15 \mathrm{~km}$, while for profile 2, it must exceed $6.14 \mathrm{~km}$ (and these layers must occur in environments with 20 $25 \mathrm{~m} \mathrm{~s}^{-1}$ horizontal winds). We were also unable to find 

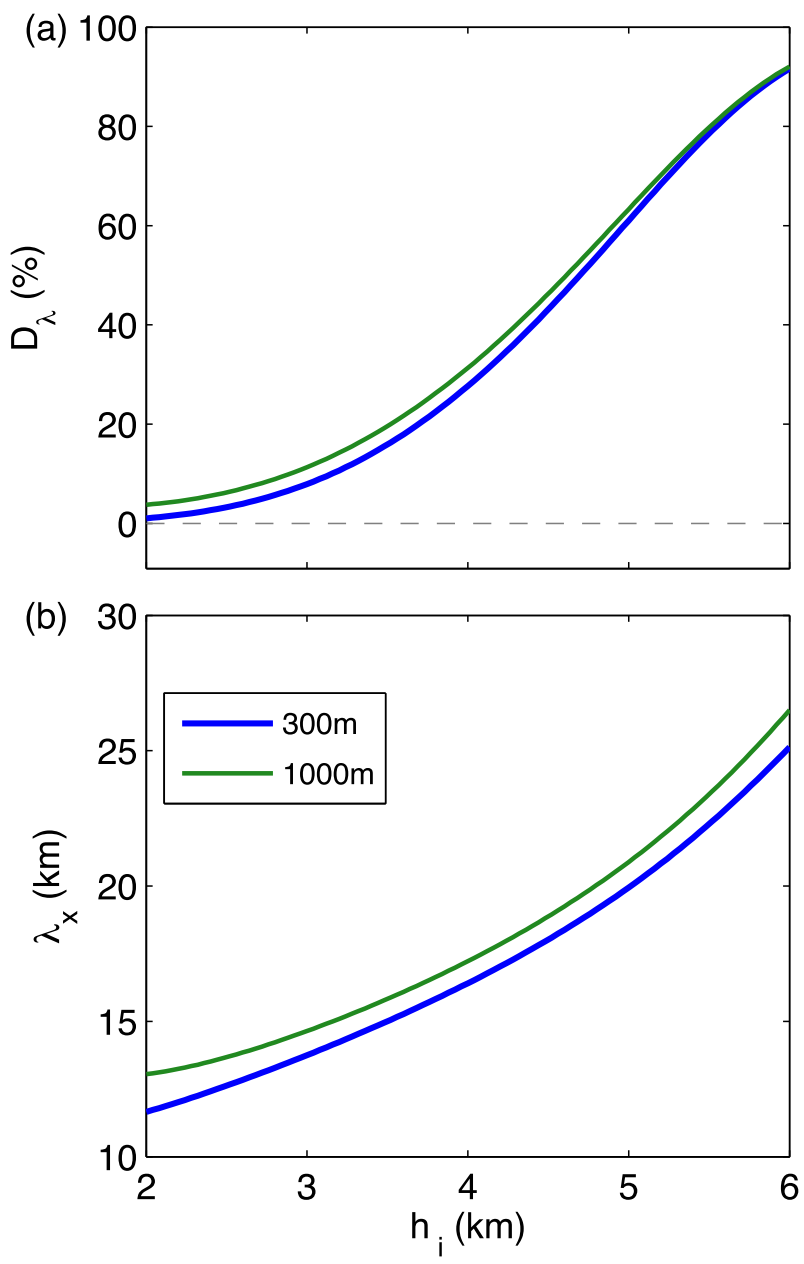

FIG. 12. Influence of the height of the elevated stable layer in profile 3 on (a) downstream decay per wavelength and (b) resonant wavelength. Blue (green) curves are for a 300 (1000)-m thick inversion. Height is measured between the surface and the top of the layer.

a variant of the more realistic profile 3 that could support an $M=2$-type mode without relying on an unrealistically deep elevated inversion.

Nevertheless, one additional factor that may reduce the chances of observing $M=2$ modes is that for a given environmental profile, they have longer horizontal wavelengths than the $M=1$ modes and thus tend to leak energy more rapidly into the stratosphere. An example is shown in Fig. 16, which shows environmental profiles for two cases similar to those in Fig. 3, except that the lowest layer is $6.5 \mathrm{~km}$ deep and the $M=2$ modal structure is plotted in Figs. 16b,c. When a stratosphere is present (with $h_{s}=10 \mathrm{~km}$ and $N_{s}=0.02 \mathrm{~s}^{-1}$ ), the wavelength increases substantially and there is significant leakage into the stratosphere. Vertical cross sections of $w$ are shown for this same case in Fig. 17. The nodal line is clearly visible at an elevation of about $4 \mathrm{~km}$. Also evident is the rapid downstream decay when the stratosphere is present.

\section{Conclusions}

We have considered the structure of trapped lee-wave modes that leak energy upward through a layer representative of the stratosphere. We obtained the counterintuitive result that such modes grow with height in the stratosphere. The physical reason for this growth is that when trapped waves that decay downstream impinge on the tropopause, the wave packets launched nearest the source contain larger-amplitude waves, and as they propagate upward and downstream parallel to the group velocity vector, they wind up above packets of weaker waves that enter the stratosphere farther downstream (see the schematic in Fig. 1). Nevertheless, the wave amplitude in the decaying trapped wave train drops to zero above some threshold roughly determined by a line parallel to the group velocity passing through the top of the source region. Confirmation that these modes are physically realizable comes from comparisons with steady-state solutions computed beginning with an initially horizontal flow using a time-dependent numerical model of the Boussinesq governing equations. Our modes are in good quantitative agreement with these steady-state numerical solutions (Fig. 6).

The most fundamental behavior common to all the cases we tested is that the rate of leakage into the stratosphere increases as a larger fraction of the maximum low-level wave amplitude penetrates upward to the tropopause. All other factors being equal, the downstream dissipation of trapped waves is reduced as the height of the tropopause increases because there is a deeper layer throughout which the partially trapped mode will decay before upward propagation begins. Our results show the rate of downstream decay is much more sensitive to tropopause height than to the stratospheric values of $N / U$ provided those $N / U$ are at least a little larger than the minimum value required to support upward propagation.

Lee waves with short horizontal wavelengths tend to experience less leakage than longer wavelength modes. This is primarily because the vertical scale height over which short waves decay tends to be shorter than that for longer waves. If $N$ and $U$ are constant in the upper troposphere, (6) gives the $e$-folding scale for such decay as

$$
\left[\left(\frac{2 \pi}{\lambda_{x}}\right)^{2}-l^{2}\right]^{-1 / 2}
$$

which decreases as $\lambda_{x}$ decreases. Short horizontal wavelengths also make it more likely that the mode can 
a)

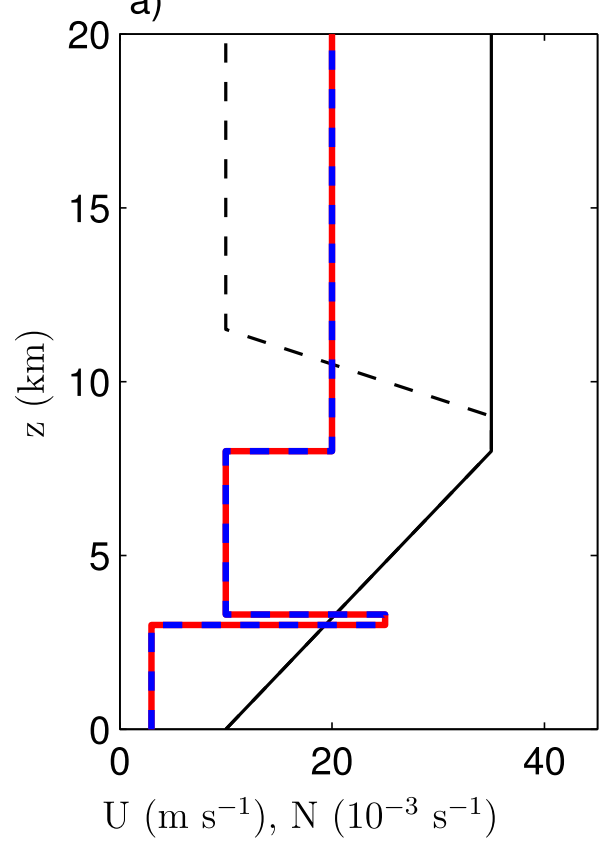

b) $\mathrm{h}_{s}=8.0 \mathrm{~km}$

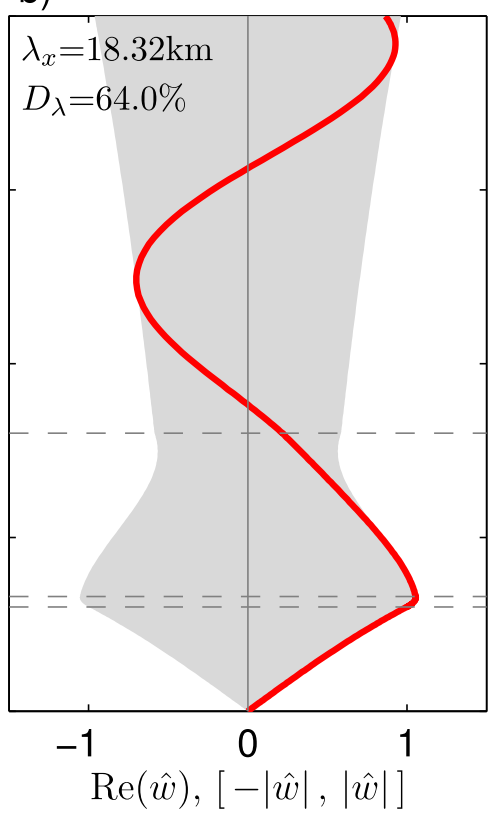

c) $\mathrm{h}_{s}=8.0 \mathrm{~km}$

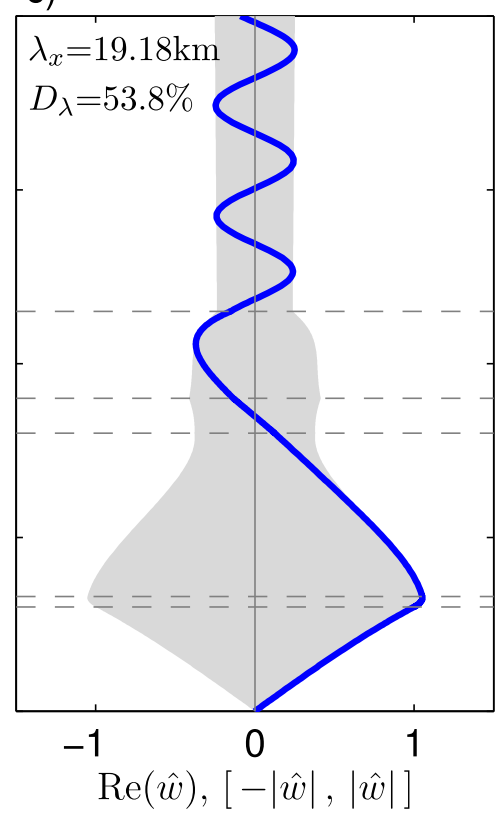

FIG. 13. As in Fig. 8, except $N_{s}=0.02 \mathrm{~s}^{-1}$ with (b) $h_{s}=8 \mathrm{~km}$, and (c) there is profile-4 reverse shear in the lower stratosphere. The top and bottom of the shear layer is indicated by the uppermost pair of thin dashed lines in (c) and the wind speed in that layer is plotted as a function of height by the black dashed line in (a).

be completely trapped, which will be the case if $\lambda_{x}<$ $2 \pi / l_{\text {top }}$, where $l_{\text {top }}$ is the Scorer parameter in top stratospheric layer where $N$ and $U$ are uniform.

The rate at which a lee-wave train damps downstream due to stratospheric leakage is highly dependent on the details of the atmospheric structure. Profile 3, which is representative of the actual atmospheric structures that trap lee waves (including an elevated inversion and tropospheric wind shear) supports waves with $\lambda_{x} \approx$ $16 \mathrm{~km}$ that undergo strong downstream leakage when the tropopause height is $8.5 \mathrm{~km}$ and more moderate leakage when $h_{s}$ increases to $10 \mathrm{~km}$. Adding a layer of realistic reversed shear in the lower stratosphere has little impact on the behavior of profile- 3 modes. Two other simple constant $U$ profiles in which a stratosphere is added to two-layer tropospheric structures where $N$ is (a) $\mathrm{h}_{\mathrm{s}}=8.0 \mathrm{~km}$

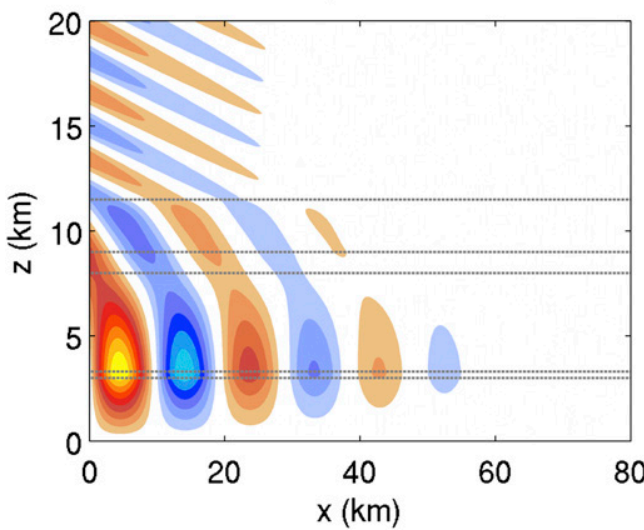

(b) $h_{s}=10.0 \mathrm{~km}$

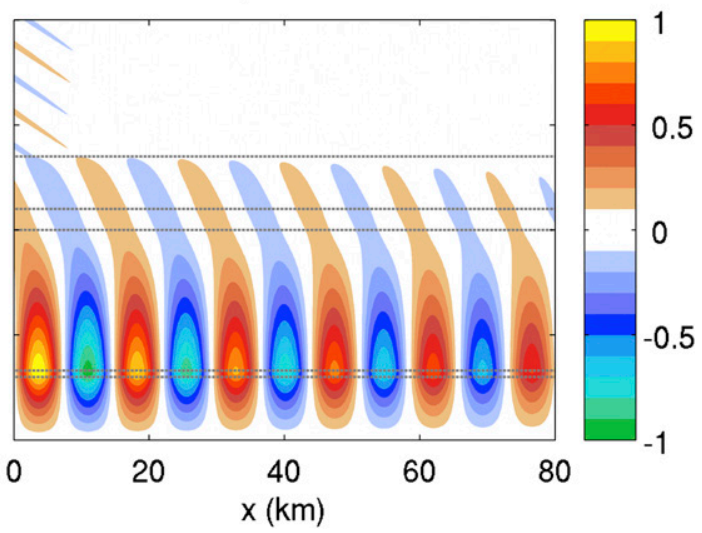

FIG. 14. Contours of $w$ in a vertical cross section for modes supported by profile 4 with $h_{s}$ at (a) 8 and (b) $10 \mathrm{~km}$. Contours have an interval of 0.1 ; thin dashed gray lines indicate, in ascending order, the locations of the inversion, the tropopause, and the stratospheric layer of reversed shear. 

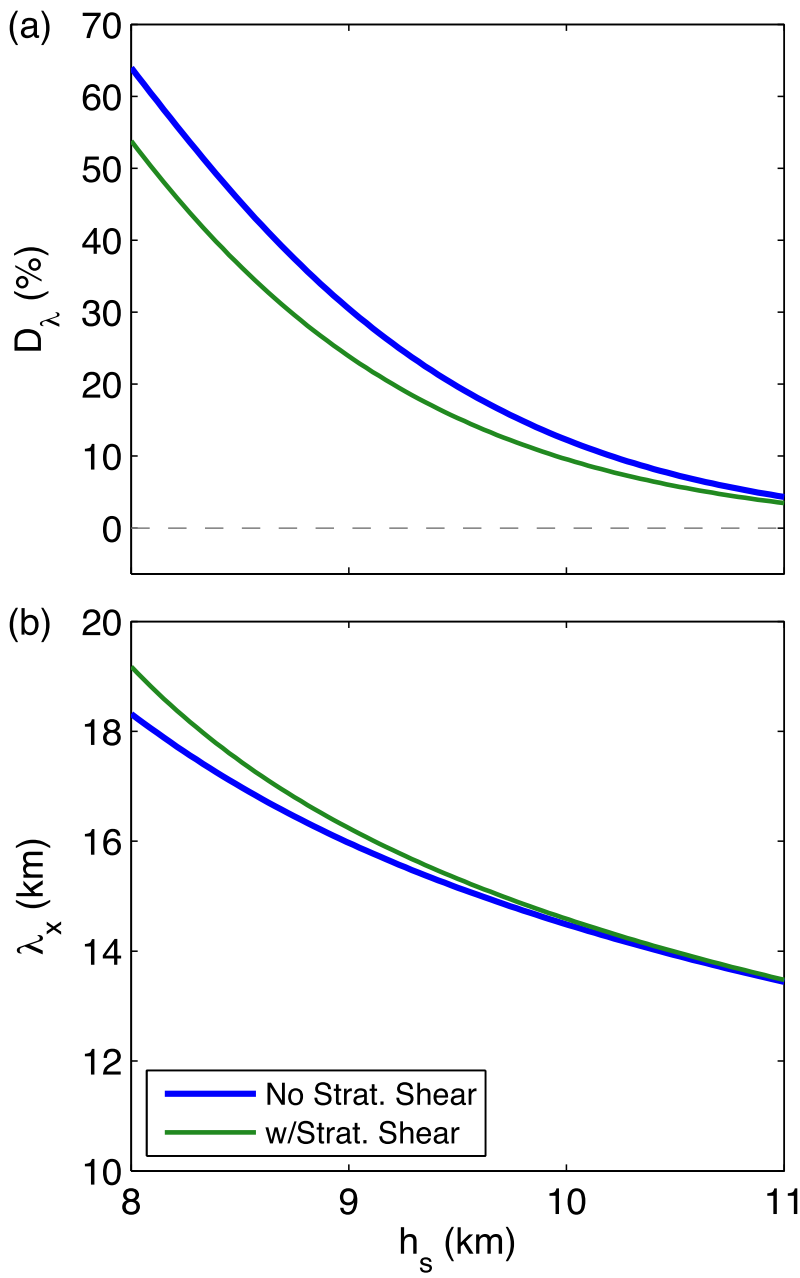

FIG. 15. Influence of stratospheric reversed shear on (a) $D_{\lambda}$ and (b) $\lambda_{x}$ as a function of tropopause height $h_{s}$. Green (blue) lines plot the results with (without) reversed shear.

constant within each layer were also considered, although neither profile is a very good representation of actual atmospheric conditions. Profile 1 , with $\lambda_{x} \approx 9 \mathrm{~km}$, is almost unaffected by the presence of the stratosphere, whereas profile 2 , with $\lambda_{x} \approx 21 \mathrm{~km}$, does experience significant leakage.

Other processes, such as boundary layer dissipation or the lateral dispersion of energy from a three-dimensional ridge of limited length, can also be responsible for the downstream decay of trapped mountain lee waves. Indeed, profile 1 was used by Jiang et al. (2006) to study the dissipation of lee waves by boundary layer processes. In Part II, we examine the relative importance of boundary layer processes and stratospheric leakage in the dissipation of trapped mountain lee waves.

Acknowledgments. The manuscript benefited from comments by two anonymous reviewers. This research was supported by National Science Foundation Grant AGS-1138977.

\section{APPENDIX}

\section{The Numerical Algorithm}

Equation (3) is a boundary value problem in which the horizontal wavenumbers $k$ are the eigenvalues and the $\hat{w}(z)$ are eigenfunctions. This problem requires the specification of two boundary conditions. At the surface downstream of the mountain, the lower boundary condition is $\hat{w}(0)=0$. At the top of the domain, assuming $k_{r}>0$, we apply

$$
\left.\frac{d \hat{w}}{d z}\right|_{z_{\text {top }}}-i m_{\text {top }} \hat{w}\left(z_{\text {top }}\right)=0,
$$

where $m_{\mathrm{top}}=\left(l_{\mathrm{top}}^{2}-k^{2}\right)^{1 / 2}$ and the positive square root is selected as per the discussion in section 2. Here the subscript "top" denotes values at the top of the numerical domain. Our formulation assumes $N$ and $U$ are uniform through the topmost layer. The condition (A1) fails for $k=l_{\text {top }}$, for which the resulting eigenfunctions are linear in $z$ throughout the top layer. We do not consider these modes, which require $\hat{w}(z)=0$ in the top layer for the solution to remain bounded with height.

The specification of (A1) makes the discretized boundary value problem implicit. We solve it using an algorithm with two conceptual steps. First, given an arbitrary value of $k$ (not necessarily an eigenvalue), we find separate solutions within upper and lower subdomains, each of which satisfies all appropriate boundary and matching conditions except that for $d \hat{w} / d z$ at the boundary between the two subdomains. Then $k$ is iteratively adjusted using a shooting technique to converge to the true eigenvalue that satisfies the correct matching condition on $d \hat{w} / d z$. The details of these two parts of the algorithm are described below. A set of MATLAB functions that implement this method will be made available at http://www. atmos.washington.edu/ bloss/TrappedWavesMATLAB after the publication of this paper.

\section{a. Solution for arbitrary $k$ within each subdomain}

Although our method is fully applicable to profiles with smooth variations in the Scorer parameter, in this paper, we only consider vertical profiles of stability $N(z)$ that are piecewise constant and wind profiles $U(z)$ that are piecewise linear. Because the Scorer parameter $l(z)$ is singular at jumps in $d U / d z$, the domain is decomposed into layers $H_{i}<z<H_{i+1}$ for $i=0, \ldots, N-1$ with the surface at $z=H_{0}$ and the top of the domain at $z=H_{N}$ (generally $20 \mathrm{~km}$ in the present work). Within each 

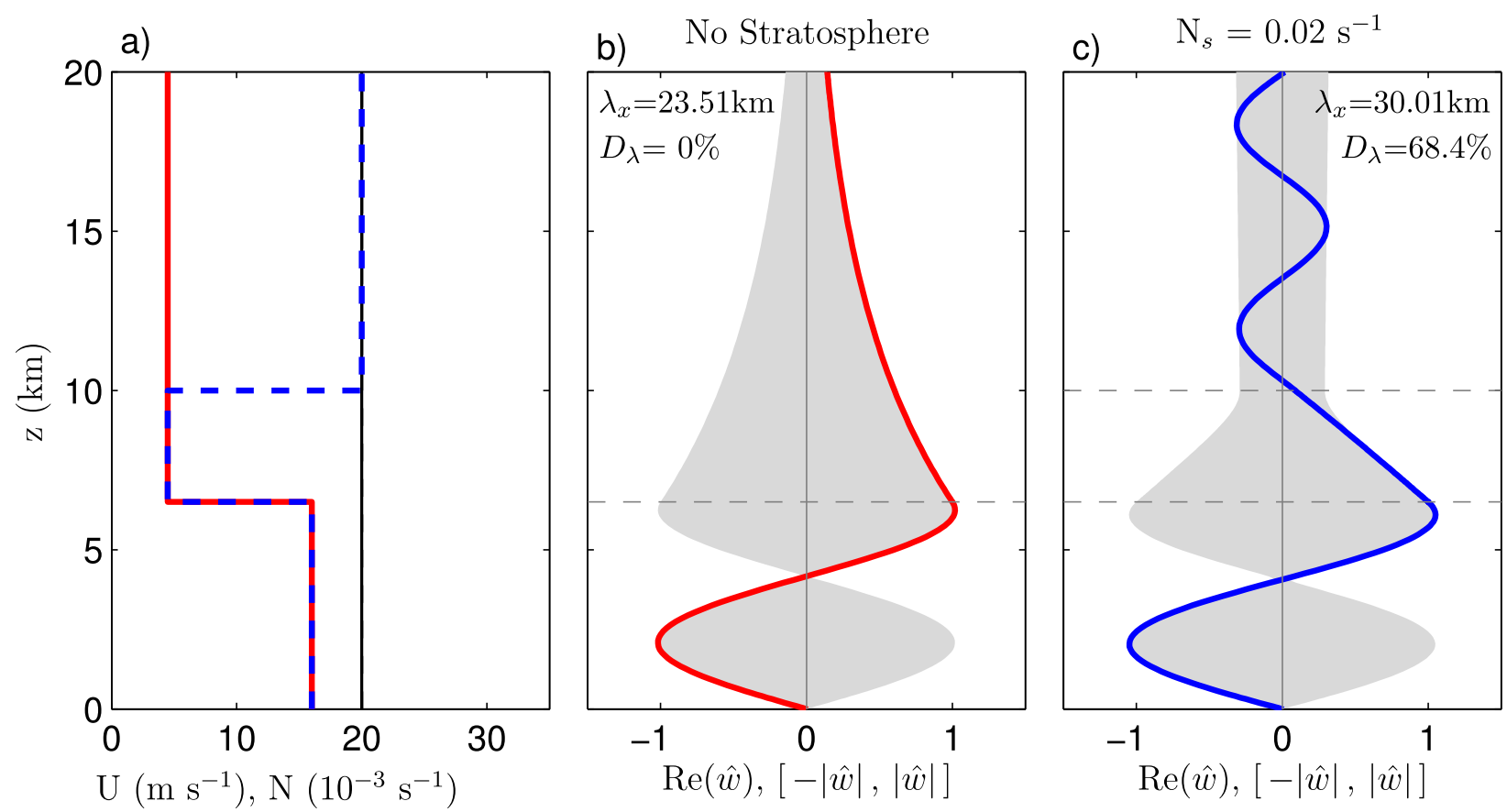

FIG. 16. Vertical structures for the same case shown in Fig. 3, except these are $M=2$ modes and the lowest layer is $6.5 \mathrm{~km}$ deep.

layer, (3) is discretized using a Chebyshev collocation technique (Trefethen 2000). The top and lower boundaries and the interfaces between layers (i.e., $z=H_{i}$ for $i=0, \ldots, N)$ are included in the discretization, but the governing equation (3) is not enforced at those locations. Rather, the boundary conditions are enforced at the surface and the top of the domain, and matching conditions are applied at the interface between the internal layers. The kinematic matching condition requires $\hat{w}$ to be continuous across the interface. The dynamic boundary condition requires pressure to be continuous across the interface, which, using the linearized horizontal momentum and the continuity equations, yields the condition

$$
-\left.U^{+} \frac{d \hat{w}}{d z}\right|^{+}+\left.\hat{w}^{+} \frac{d U}{d z}\right|^{+}=-\left.U^{-} \frac{d \hat{w}}{d z}\right|^{-}+\left.\hat{w}^{-} \frac{d U}{d z}\right|^{-}
$$

here the superscripts + and - represent the values just above and below the interface. Using the continuity of $\hat{w}$ (a) No Stratosphere

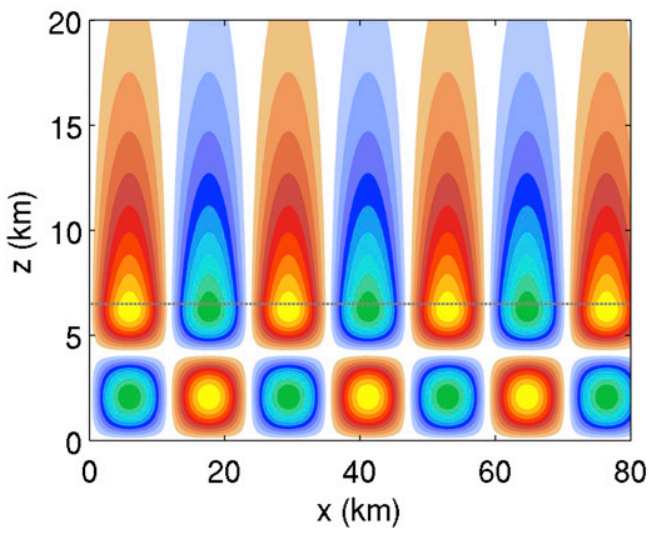

(b) $h_{s}=10 \mathrm{~km}, N_{s}=0.02 \mathrm{~s}^{-1}$

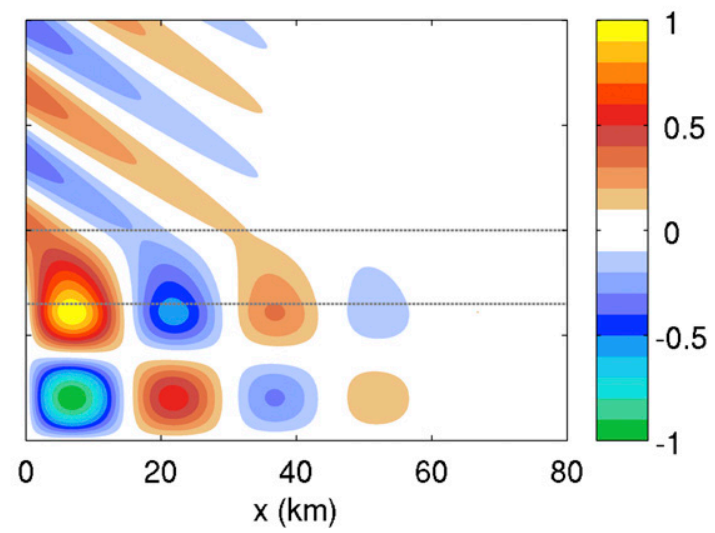

FIG. 17. Contours of $w$ in a vertical cross section for $M=2$ modes supported by the environmental profile plotted in Fig. 16a: (a) without and (b) with a stratosphere. Contours have an interval of 0.1 ; thin dashed gray lines indicate the top of the lower layer and, in (b), the tropopause. 
across the interface and assuming $U$ is also continuous, (A2) reduces to

$$
\left.\frac{d \hat{w}}{d z}\right|^{+}-\left.\frac{d \hat{w}}{d z}\right|^{-}=\left(\left.\frac{d U}{d z}\right|^{+}-\left.\frac{d U}{d z}\right|^{-}\right) \frac{\hat{w}}{U}
$$

The derivative $d \hat{w} /\left.d z\right|^{+}$is computed using the Chebyshev differentiation matrix for the layer above the interface and depends on the values of $\hat{w}$ only within that layer and at the interfaces above and below that layer; $d \hat{w} /\left.d z\right|^{-}$is computed similarly. The discretized version of (A3) is enforced for $z=H_{i}$ with $i=1, \ldots, N-1$.

The system described above will only have solutions for values of $k$ which are eigenvalues of the full system. In implementing a shooting technique, the matching condition (A3) at the top of the lowest layer $z=H_{1}$ is not enforced, and solutions are found in the subdomains, $0 \leq z \leq H_{1}$ and $H_{1} \leq z \leq H_{N}$, with the boundary condition $\hat{w}\left(z=H_{1}\right)=1$ enforced on both solutions. As the amplitude of the eigenfunction $\hat{w}(z)$ is arbitrary, the only additional requirement imposed by this condition is that $H_{1}$ is not a node of the solution. This has not been an issue in the present work, but, if there were a node at this location, one could break the domain in another location as necessary.

\section{b. Shooting technique}

Given an initial guess for $k$, solutions to (3) may be found in the subdomains $\left[0, H_{1}\right]$ and $\left[H_{1}, H_{N}\right]$, but, in general, the matching condition (A3) will not be satisfied at $z=H_{1}$. The MATLAB function fminsearch is employed to minimize the residual of (A3) at $z=H_{1}$. If the residual is smaller than $10^{-8}$, it is accepted as a valid solution. As an aid to obtaining a good initial guess for $k$, we plotted the absolute value of the residual as a function of $k$, both along the real axis and in the complex plane using relatively coarse resolution. Initial guesses for $k$ were selected at points where the residual was small.

\section{REFERENCES}

Berkshire, F. H., 1975: Two-dimensional linear lee wave modes for models including a stratosphere. Quart. J. Roy. Meteor. Soc., 101, 259-266, doi:10.1002/qj.49710142809.

—_, and F. W. G. Warren, 1970: Some aspects of linear lee wave theory for the stratosphere. Quart. J. Roy. Meteor. Soc., 96, 5066, doi:10.1002/qj.49709640707.

— lee waves in an atmosphere of three layers. Quart. J. Roy. Meteor. Soc., 104, 895-909, doi:10.1002/qj.49710444204.

Brown, P. R. A., 1983: Aircraft measurements of mountain waves and their associated momentum flux over the British Isles.
Quart. J. Roy. Meteor. Soc., 109, 849-865, doi:10.1002/ qj.49710946211.

Corby, G. A., and J. S. Sawyer, 1958: The air flow over a ridge-The effects of the upper boundary and high-level conditions. Quart. J. Roy. Meteor. Soc., 84, 25-37, doi:10.1002/ qj. 49708435904 .

Doyle, J. D., and D. R. Durran, 2002: The dynamics of mountainwave-induced rotors. J. Atmos. Sci., 59, 186-201, doi:10.1175/ 1520-0469(2002)059<0186:TDOMWI >2.0.CO;2.

Georgelin, M., and F. Lott, 2001: On the transfer of momentum by trapped lee waves: Case of the IOP 3 of PYREX. J. Atmos. Sci., 58, 3563-3580, doi:10.1175/1520-0469(2001)058<3563: OTTOMB $>2.0 . \mathrm{CO} ; 2$.

Gill, A. E., 1982: Atmosphere-Ocean Dynamics. International Geophysics Series, Vol. 30, Academic Press, 662 pp.

Jiang, Q., J. D. Doyle, and R. B. Smith, 2006: Interaction between trapped waves and boundary layers. J. Atmos. Sci., 63, 617633, doi:10.1175/JAS3640.1.

Keller, T. L., 1994: Implications of the hydrostatic assumption on atmospheric gravity waves. J. Atmos. Sci., 51, 1915-1929, doi:10.1175/1520-0469(1994)051<1915:IOTHAO > 2.0.CO;2.

Nance, L. B., and D. R. Durran, 1997: A modeling study of nonstationary trapped mountain lee waves. Part I: Meanflow variability. J. Atmos. Sci., 54, 2275-2291, doi:10.1175/ 1520-0469(1997)054<2275:AMSONT>2.0.CO;2.

Queney, P., G. Corby, N. Gerbier, H. Koschmieder, and J. Zierep, 1960: The airflow over mountains. WMO Tech. Rep. 34, 135 pp.

Ralph, F., P. Neiman, T. Keller, D. Levinson, and L. Fedor, 1997: Observations, simulations, and analysis of nonstationary trapped lee waves. J. Atmos. Sci., 54, 1308-1333, doi:10.1175/ 1520-0469(1997)054<1308:OSAAON>2.0.CO;2.

Scorer, R., 1949: Theory of waves in the lee of mountains. Quart. J. Roy. Meteor. Soc., 75, 41-56, doi:10.1002/ qj. 49707532308 .

Shutts, G., 1992: Observations and numerical-model simulation of a partially trapped lee wave over the Welsh mountains. Mon. Wea. Rev., 120, 2056-2066, doi:10.1175/1520-0493(1992)120<2056: OANMSO $>2.0 . \mathrm{CO} ; 2$.

Smith, R. B., 1976: The generation of lee waves by the Blue Ridge. J. Atmos. Sci., 33, 507-519, doi:10.1175/1520-0469(1976)033<0507: TGOLWB $>2.0 . C O ; 2$.

- S. Skubis, J. D. Doyle, A. S. Broad, C. Kiemle, and H. Volkert, 2002: Mountain waves over Mont Blanc: Influence of a stagnant boundary layer. J. Atmos. Sci., 59, 2073-2092, doi:10.1175/ 1520-0469(2002)059<2073:MWOMBI >2.0.CO;2.

—, Q. Jiang, and J. D. Doyle, 2006: A theory of gravity wave absorption by a boundary layer. J. Atmos. Sci., 63, 774-781, doi:10.1175/JAS3631.1.

Tannhauser, D., and N. Moiseyev, 1995: The solution of lee wave problems by the complex coordinate method. Meteor. Z., 4, 257-265.

Trefethen, L. N., 2000: Spectral Methods in MATLAB. SIAM, $165 \mathrm{pp}$.

Vergeiner, I., 1971: An operational linear lee wave model for arbitrary basic flow and two-dimensional topography. Quart. J. Roy. Meteor. Soc., 97, 30-60, doi:10.1002/qj.49709741104.

Wurtele, M. G., R. D. Sharman, and T. L. Keller, 1987: Analysis and simulations of a troposphere-stratosphere gravity wave model. Part I. J. Atmos. Sci., 44, 3269-3281, doi:10.1175/ 1520-0469(1987)044<3269:AASOAT>2.0.CO;2. 